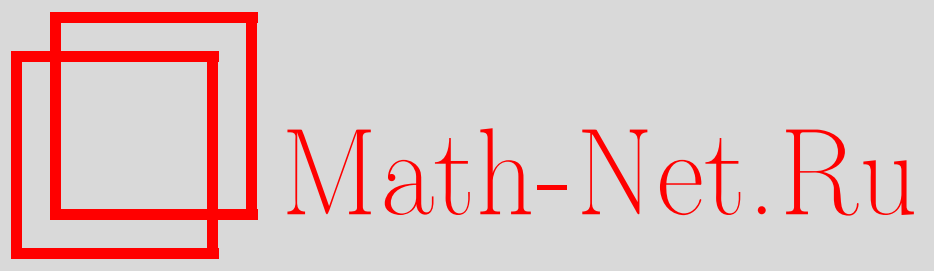

В. П. Маслов, Термодинамика флюидов, закон перераспределения энергий, двумерный конденсат и $T$ отображение, $T M \Phi, 2009$, том 161, номер 3, 420-458

DOI: https://doi.org/10.4213/tmf6451

Использование Общероссийского математического портала Math-Net.Ru подразумевает, что вы прочитали и согласны с пользовательским соглашением http://www . mathnet.ru/rus/agreement

Параметры загрузки:

IP: 3.89 .197 .203

26 апреля 2023 г., $15: 25: 48$

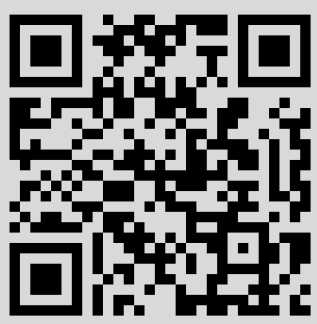




\title{
ФИЗИКА
}

Том 161, № 3

декабрь, 2009

2009 г.

В. П. Маслов*

\section{ТЕРМОДИНАМИКА ФЛЮИДОВ, ЗАКОН ПЕРЕРАСПРЕДЕЛЕНИЯ ЭНЕРГИЙ, ДВУМЕРНЫЙ КОНДЕНСАТ И Т-ОТОБРАЖЕНИЕ}

\begin{abstract}
Подробно рассмотрено совпадение экспериментальных данных с результатами расчетов по формулам, полученным автором ранее для реального газа. Сформулирован закон перераспределения энергий как следствие возникновения димеров. Приведена теорема о двумерном конденсате для любого газа. Описан процесс перехода к равновесной термодинамике при увеличении давления поршнем.
\end{abstract}

Ключевые слова: димеры, кластеры, парастатистика, конденсат, фрактальная размерность, флюиды, термодинамика, теория распределений, теория чисел.

\section{1. ЗАКОН ПЕРЕРАСПРЕДЕЛЕНИЯ ЭНЕРГИЙ}

В предыдущей работе [1] рассматривался случай идеальных флюидов, и наличие ловушки в задаче рассеяния являлось физическим аргументом в пользу того, что явление конденсата в парастатистическом распределении приводит к образованию не аналога бозе-конденсата, а димеров.

Раньше вместо термина "димеры" использовалось выражение "ассоциация молекул". 103 года тому назад, в 1906 году великий термодинамик Ван дер Ваальс в докладе Амстердамской Академии наук сообил, что только предположение об "ассоциации молекул" может объяснить отклонение экспериментальных данных от его уравнения состояния и позволяет установить соотношение, удовлетворяющее экспериментальным данным. Однако если мы переходим к реальному газу и флюидам, то нужно помнить, что слова "мономеры" и “димеры" - это только метафоры. Они имеют прямое значение лишь для идеального (в понимании автора, см. [1]) газа. Речь идет о том, что вместо закона равнораспределения по энергиям мы получаем закон перераспределения энергий. Если мы предполагаем, что на изотерме $T=T_{\text {cr }}$ в исходном газе при малом давлении и большом объеме имеет место закон равнораспределения, то это с точки зрения нашей метафоры - мономеры.

Фазовый переход при $P=P_{\text {сг }}$ от точки $V / R=0.444 \mathrm{~cm}^{3} /$ моль к точке $V / R=$ $0.296 \mathrm{~cm}^{3} /$ моль означает перераспределение энергий, при котором поступательная

* Московский государственный университет им. М. В. Ломоносова, Москва, Россия. E-mail: v.p.maslov@mail.ru 


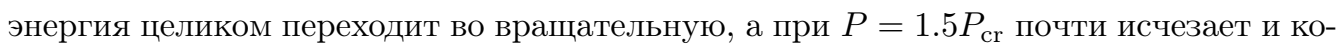
лебательная энергия, переходя во вращательную, флюид становится несжимаемым на изотерме

$$
Z=\frac{P V}{R T} \quad \text { при } \quad T=1, \quad P>2 .
$$

На рис. 4 работы [1] (см. также рис. 3 настоящей работы) функция $Z(P)(1)$ почти линейна, это означает, что $V=$ const, т.е. несжимаемость, колебания при таком давлении почти исчезают, а значит, скорость звука во флюиде очень велика. Таким образом, фазовый переход, обозначенный вертикальным отрезком на указанном рисунке, есть перераспределение энергии: поступательная энергия $P V$ при постоянной критической температуре превращается во вращательную энергию, уменьшившись на $2 P V / 3=2 / 3 \cdot 0.444=0.296$. Можно условно считать, что это аналог перехода газ-жидкость во флюиде.

Однако переход газ-жидкость на горизонтальном отрезке Максвелла в некотором смысле устойчив. Наличие примесей аэрозолей, как правило, только препятствует метастабильному состоянию и приближает переход Гиббса, Максвелла, Ван дер Ваальса - фазовый переход 1-го рода. В рассматриваемом нами случае наличие небольшой, например в 1\%, примеси бензола в углекислом газе в критической точке существенно меняет его свойства, приближая по некоторым свойствам к бензолу. При неравновесном увеличении давления возникает конвекция вихревого характера, так что даже можно говорить о переходе хаотического движения в упорядоченное когерентное вихревое движение (самоорганизацию), которое, правда, после возникновения колмогоровской изотропной турбулентности затухает и система возвращается к равновесной термодинамической картине (см. рис. 4 из [1] ${ }^{1)}$ ).

Согласно предъявленной концепции трехмерных кластеров, т.е. кластеров, содержащих молекулы, окруженные со всех сторон другими молекулами (зародышей капли), не возникает. Мы полагаем, что дисперсной среды не должно быть, что отличается от мнения, высказанного некоторыми крупными химиками, о том, что дисперсная среда возникает всегда. Трехмерные кластеры, т.е. кластеры, содержащие хотя бы одну молекулу, окруженную другими, возникают при $T<T_{\mathrm{cr}}$. Мы назовем такие кластеры, по аналогии с математическим термином ${ }^{2)}$, доменами.

Согласно математической концепции автора мицеллы, как и домены, должны образовываться лишь при $T<T_{\mathrm{cr}}$. Однако экспериментальные подтверждения этому автору неизвестны.

Специалисты по молекулярной физике в своих рассуждениях обычно исходят из симметрии среднего движения молекул по всем шести направлениям. Мы применили к задаче рассеяния обычный для молекулярной физики принцип симметрии по

\footnotetext{
1) Если говорить об эконофизике, то согласно теоремам автора именно эта надкритическая ситуация - явление фазовых переходов энергии одного типа в энергию другого типа и самоорганизация

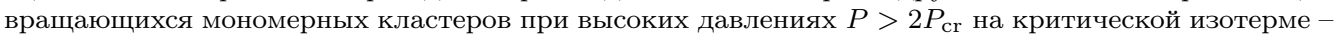
более всего подходит к описанию экономических кризисов, революции и самоорганизации, а затем турбулизации этих процессов. Причем наиболее характерно поведение углекислого газа вытянутой молекулы, небольшой изгиб которой приводит к явлению поляризации. Добавление $1 \%$ метанола в критической изотерме $\mathrm{CO}_{2}$ приводит к полной поляризации молекулы $\mathrm{CO}_{2}$ в том смысле, что $\mathrm{CO}_{2}$ в этом состоянии в ряде процессов экстракции ведет себя как $100 \%$ метанол.

2) В математике домен - это открытая область, содержащая хотя бы одну точку.
} 


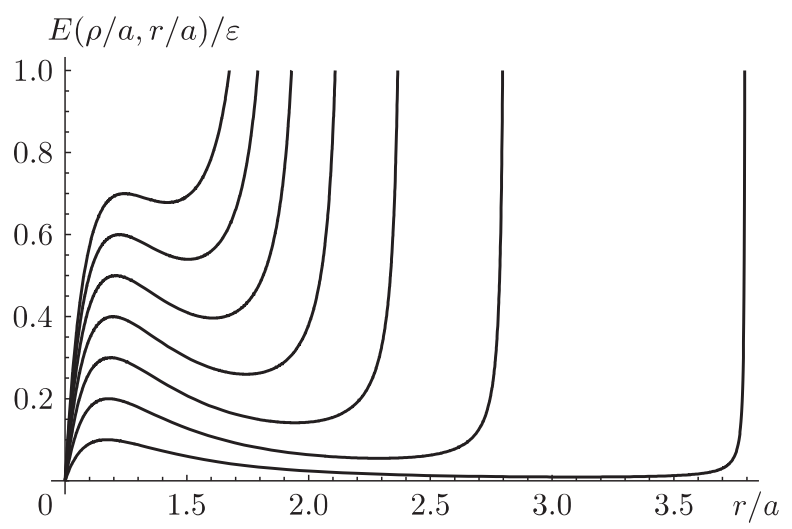

Рис. 1. Значения $E(\rho, r)=4 \varepsilon a^{6}\left(\frac{a^{6}}{r^{6}}-\frac{a^{12}}{r^{12}}\right)\left(\frac{\rho^{2}}{r^{2}}-1\right)^{-1}$ для различных значений прицельного параметра.

всем направлениям, но не для определения длины свободного пробега, а других величин молекулярной физики. Следовательно, навстречу друг другу движется 1/12 часть всех частиц. Таких направлений три, поэтому сталкивается $1 / 4$ часть всех молекул. Их средняя энергия $k T=4 \varepsilon E$, где $k$ - константа Больцмана, $\varepsilon-$ константа в потенциале Леннарда-Джонса, $E$ - энергия пары $\left(p_{1}-p_{2}\right)^{2} / m$, где $p_{1}, p_{2}-$ импульсы двух рассеивающихся друг на друге частиц.

Впервые эти соображения, безусловно, имел в виду Больцман при выводе уравнения Больцмана и интеграла столкновения, когда он рассматривал столкновение только двух молекул. Довел их до точной математической формулировки Боголюбов в случае общего потенциала взаимодействия. Однако точной формулировки принципа симметрии для задачи рассеяния у них нет. Для них этот принцип, по-видимому, был настолько очевиден (как и для других физиков), что они его не упомянули. Эта концепция определяет соотношение лишь в среднем, однако подобно длине свободного пробега дает достаточно точное совпадение с экспериментом.

При уменьшении температуры высота барьера возрастает до величины $E=0.286 \varepsilon$, а далее начинает убывать (см. рис. 1). Согласно грубым энергетическим соображениям [2] должен образовываться дополнительный барьер при образовании самих кластеров. Этим барьером для углекислого газа могут быть мицеллы, а для нейтральных газов и метана - зародыши капли, т.е. трехмерные кластеры, у которых существует хотя бы одна молекула, окруженная другими молекулами, - прообраз капли.

За "бесконечное" время полная энергия внутри и вне ямки выравнивается. Следовательно, если из энергии мономеров вычесть энергию димеров, то получится энергия, отвечающая дну ямки (ср. [1]). Она равна $P V$ (с точностью до константы, учитывающей симметрию задач молекулярной физики), где $P$ - давление мономеров, так как в приведенной задаче рассеяния пары, попавшие в ямку, на стенку сосуда не оказывают давления.

Отношение полной энергии $E_{\max }$ к энергии, отвечающей $P V$, т.е. к $E_{\min }$, где $E_{\min }-$ дно ямки, есть величина, обратная фактору сжимаемости. Значит, $E_{\min } / E_{\max }$ 


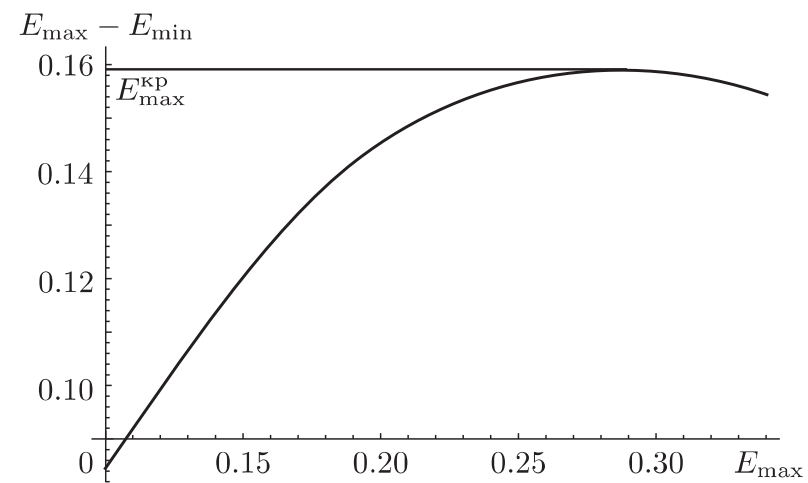

Рис. 2. График зависимости глубины ямки от выбора уровня энергии, $E_{\max }$. Максимальная глубина ямки равна 0.15899 при исходной энергии 0.286 .

есть фактор сжимаемости $Z=P V / R T$. Если мы рассматриваем фактор сжимаемости как функцию от $E_{\min }$, то это изохора при некотором фиксированном объеме. Если $T=T_{\text {cr }}\left(E=E_{\text {cr }}\right.$, см. рис. 2$)$ и $P=P_{\text {cr }}$, то $V / R=0.444$.

Димер в классической области может образовываться, если рассеивающаяся пара имеет энергию, равную высоте барьера, проскальзывая за "бесконечное" время в ямку и в силу явления вязкости, а следовательно, небольшой потери энергии, застревая в ней, поскольку на обратном пути эта пара частиц из-за потери энергии натыкается на барьер. Если пара частиц прошла выше этой точки, то вязкости и диссипации может не хватить для того, чтобы пара застряла: отразившись, эта пара пройдет обратно выше барьера. Поэтому только наличие точки $E=E_{\max }$ плюс бесконечно малая величина, где $E_{\max }$ - верхняя точка барьера, есть необходимое

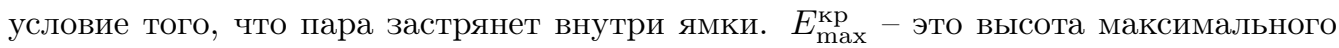
барьера (см. рис. 2).

Приведем таблицу сравнения.

\begin{tabular}{|c|c|c|c|}
\hline Вещество & $\varepsilon, \mathrm{K}$ & $T_{\mathrm{cr}} / 4$ & $E_{\text {cr }} \cdot \varepsilon / k$ \\
\hline $\mathrm{Ne}$ & 36.3 & 11 & 10.5 \\
\hline $\mathrm{Ar}$ & 119.3 & 37 & 35 \\
\hline $\mathrm{Kr}$ & 171 & 52 & 50 \\
\hline $\mathrm{N}_{2}$ & 95.9 & 31 & 28 \\
\hline $\mathrm{CH}_{4}$ & 148.2 & 47 & 43 \\
\hline $\mathrm{C}_{2} \mathrm{H}_{6}$ & 243.0 & 76 & 70 \\
\hline $\mathrm{C}_{4} \mathrm{H}_{10}$ & 313.0 & 106 & 98 \\
\hline $\mathrm{H}_{2} \mathrm{~S}$ & 301 & 93 & 87 \\
\hline $\mathrm{H}_{2} \mathrm{Se}$ & 320 & 102 & 93 \\
\hline $\mathrm{PH}_{3}$ & 251.5 & 81 & 73 \\
\hline
\end{tabular}

Почему истинные значения $T_{\text {сr }}$ превышают значения, полученные из задачи рассеяния, совершенно ясно. Установление равновесия для газа в ловушке и вне ло- 
вушки происходит (как в плазме) за бесконечное время, т.е. в конце концов полная энергия и давление выравниваются внутри и вне ловушки (см. [1]). Однако в силу наличия вязкости релаксационный процесс и измерения происходят за конечное время. Следовательно, энергия падающих мономеров все же будет несколько больше энергии застрявших димеров. Так, смог, возникший вне лощины при температуре, которая отвечает энергии проникновения через окружающие лощину препятствия за конечное время, останется более концентрированным вне лощины.

Выше значения $E_{\mathrm{B}}=0.8 \varepsilon$ ловушка исчезает. При значении $0.286 \varepsilon$ глубина ловушки максимальна и отвечает $T_{\mathrm{cr}}=1.16 \varepsilon / k$. Для неона и криптона, как видно из таблицы, совпадение достаточно хорошее. Поскольку $T_{\mathrm{B}}=3.2 \varepsilon / k$, то $T_{\mathrm{B}} / T_{\mathrm{cr}}=2.7$, что соответствует известному соотношению [3].

Температура, отвечающая $4 E_{\mathrm{B}} / k$, есть температура, выше которой димеров не возникает. Эту температуру мы и называем температурой Бойля. Согласно формулам работы [1] при приближении снизу к этой точке химический потенциал по модулю стремится к бесконечности. Это означает, что для взаимодействия Леннарда-Джонса при $T>T_{\mathrm{B}}$ фактор сжимаемости $Z$ близок к единице, и, следовательно, выше этой температуры имеют место распределения Максвелла и Гиббса, и можно говорить, что парастатистическое распределение, близкое к распределению Бозе-Эйнштейна, переходит в распределение Больцмана.

Рассмотрим величину $E_{\min } / E_{\max }\left(E_{\min }\right)$ и ее производную по $E_{\min }$ в критической точке, т.е. в точке $E_{\min } / E_{\max }=0.444$, которая дает угол наклона изохоры к вертикали $P=$ const, равный $62.4^{\circ}$ (на рис. 3 это соответствует объему $V=1$, если привести оси координат к одному масштабу). Задача рассеяния рассматривается в бесконечном пространстве, но согласно нестандартному анализу бесконечности можно градуировать. Именно, градуировки мы будем делать по объему $V / R \mathrm{~cm}^{3} /$ моль. Эту градуировку можно обосновать следующим образом:

$$
\frac{E_{\min }}{E_{\max }}=Z=\frac{P V}{R T}, \quad E_{\min }=P V,
$$

и, следовательно, при увеличении $V$ масштаб по $Z$ и $E_{\min }$ уменьшается в $1 / V$ раз.

Каждая кривая $E_{\min } / E_{\max }\left(E_{\min }\right)$ представляет собой изохору, если мы ее рассматриваем на графике $Z(P)$. Когда $V$ мало, масштаб графика изохоры увеличивается. А поскольку угол в точке $T=1$, равный const $/ V$, становится большим, то изохора, масштаб которой растягивается одновременно на $1 / V$ по абсциссе и ординате, становится менее пологой.

На рис. 3 мы приблизили экспериментальную изотерму в интервале давления от $P=0.25 P_{\text {cr }}$ до $P_{\text {сr }}$ идеальным парастатистическим распределением фрактальной размерности 2.8 и провели теоретические изохоры под вычисленным углом к прямой $T=1$. Теоретические изохоры проведены между экспериментальными изохорами, отмеченными штриховыми линиями.

В каждой точке изохоры можно однозначно определить температуру, поскольку этой точке на графике отвечает точка $P$ и точка $Z=P V_{\text {isoch }} / T$, так что $T=$ $P V_{\text {isoch }} / Z$. Поскольку $Z=V / R$ при $P=1$ и $T=1$, то другие изохоры получаются из рассматриваемой нами изменением масштаба по $Z$ и $P$. 


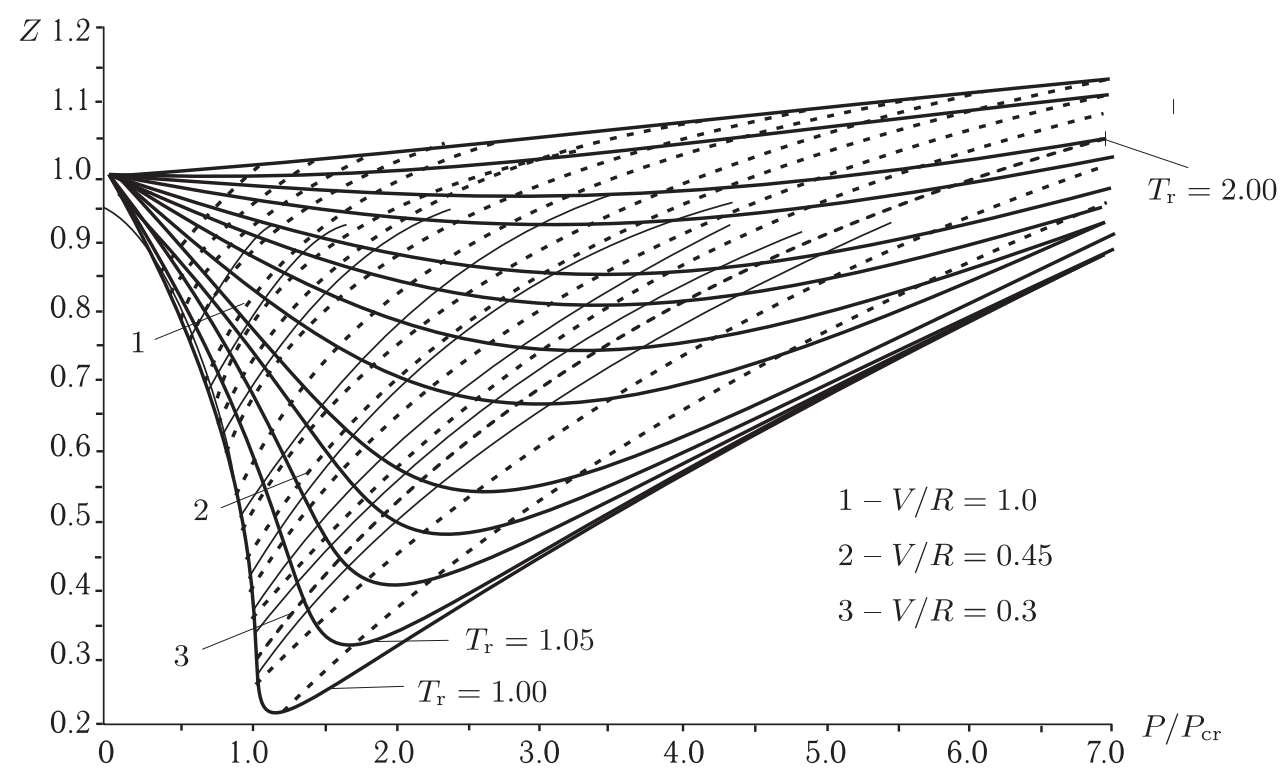

Рис. 3. Экспериментальные изохоры (жирные и штриховые линии) для для метана, этилена, этана, пропана, н-бутана, изопентана, н-гептана, азота, двуокиси углерода и воды. Теоретические изохоры и изотерма (тонкие линии) построены до $Z=0.95$.

Таким образом, для построения теоретического графика в нулевом приближении (следующие приближения см. ниже) все готово. Критическая изотерма $T=1$ проведена вплоть до точки 0.296. Углы к кривой $P \leqslant 1$, под которыми нужно провести из каждой точки изотермы $T=1$ теоретические изохоры нужного масштаба, позволяют в нулевом приближении построить график изотерм и изохор на ПлосКости $Z(P)$.

ЗАмЕчАниЕ 1. Нетрудно убедиться, что для парастатистического распределения, как и в случае идеального бозе-газа, размерность будет меняться (с точностью до тысячных знаков) от 2.4 до 2.8 на отрезке $T=T_{\mathrm{cr}}, P=P_{\mathrm{cr}}$, т.е. при $T=T_{\mathrm{cr}}$,

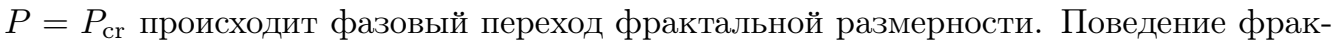
тальной размерности при $T=T_{\mathrm{cr}}, P<P_{\mathrm{cr}}$ теоретически можно вычислить, применив метод $T$-отображения к интегральному уравнению среднего поля. Однако точность экспериментов по ряду газов, указанных на рис. 3 работы [4], не позволяет провести сравнения эксперимента с теорией. Поэтому для сравнения с экспериментальными точками, взятыми из рис. 3, для размерностей $3,2.8$ и 2.4 мы приводим рис. 4.

Разумеется, наличие димеров в реальном газе уменьшает размерность 3 . Но насколько? Производная $E_{\min } / E_{\max }\left(E_{\min }\right)$ в критической точке, т.е. в точке $E_{\max }^{\text {кр }}$, отвечает углу $62.4^{\circ}$. Это постоянная величина, и, следовательно, угол изохоры по отношению к прямой $Z=$ const должен быть обратно пропорционален $V$. В результате оказывается, что совпадение с экспериментальным графиком менее точное, чем разброс, который возникает на рис. 3 для разных газов. 


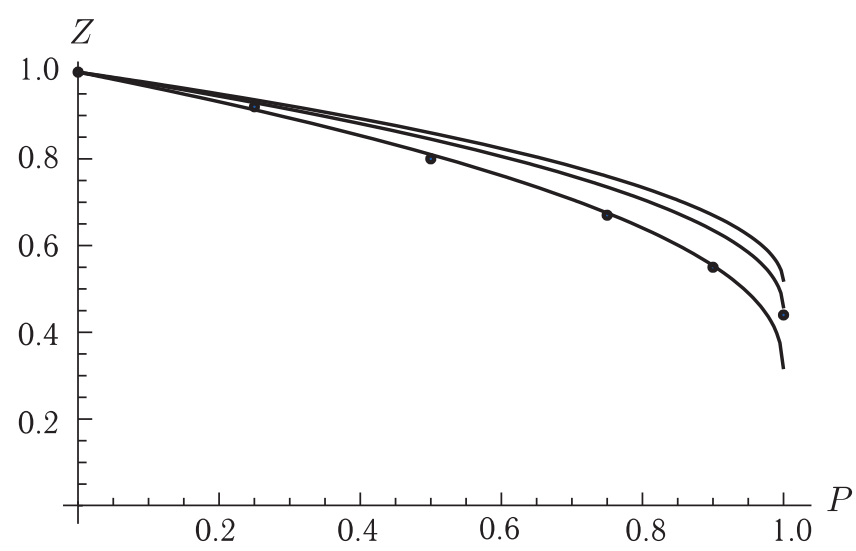

Рис. 4. Сравнение теоретических значений $Z(P)$ с экспериментальными. Кривая $\gamma=0.5$ отвечает размерности 3 , кривая $\gamma=0.4$ - размерности 2.8 , кривая $\gamma=0.2$ - размерности 2.4. Точки - экспериментальные данные, отвечающие рис. 3. Расстояние по вертикали между крайними точками кривых $\gamma=0.4$ и $\gamma=0.2$ в точке $P=1$ соответствует расстоянию между экспериментальными кривыми на рис. 3. На изотерме $d \mu=V d p=Z(\mu) / P d P$ значение $Z=\int_{0}^{\infty}\left(\varepsilon^{\gamma} d \varepsilon\right) /\left(e^{(\varepsilon-\mu) / T}-1\right) / T \int_{0}^{\infty}\left(\varepsilon^{\gamma+1} d \varepsilon\right) /\left(e^{(\varepsilon-\mu) / T}-1\right)$. Для размерности 3 адиабату Пуассона см. в монографии [5]. Аналогично адиабата для размерности 2.8 имеет вид $P V^{12 / 7}=$ const, а для размерности 2.4 имеет вид $P V^{11 / 6}=$ const.

Эксперимент, который дает значение $V=0.296$, более простой, он описан в любом учебнике по молякулярной физике. K этому ответу приходят, повышая температуру до критической, пока в заданном объеме не исчезнет, постепенно расширяясь, пленка поверхностного натяжения.

Эксперимент, который приведет к точке 0.444, более сложный. Газ должен быть нагрет до температуры $T=T_{\text {cr }}$ при низком давлении, затем поршнем нужно увеличивать давление до $P=P_{\mathrm{cr}}$. Ответ существенно зависит от пути прихода к состоянию равновесия. После достижения температуры вырождения поршень будет двигаться без повышения давления, как в бозе-газе. Такой эксперимент мне известен только для ртути (эксперимент группы академика И. К. Кикоина). Можно, наоборот, после достижения первым способом точки $V / R=0.296$ начать постепенно увеличивать объем. В этом случае при равновесном процессе увеличения объема мы дойдем до точки 0.444, а далее при медленном увеличении объема возникнет эффект, аналогичный эффекту Джоуля-Томсона, возможно, заключающийся в уменьшении фрактальной размерности (увеличении числа димеров), и далее фрактальная размерность не меняется.

Димеры играют роль пористой среды для мономеров. В эффекте Джоуля-Томсона при расширении объема через пористую среду понижается температура. При уменьшении температуры должны увеличиваться число димеров и, следовательно, фрактальная размерность мономеров. Фрактальная размерность уменьшается ровно до того значения, которое соответствует фрактальной размерности газа в опи- 
санном выше стандартном эксперименте, когда давление равно $0.9 P_{\text {сr }}$ и жидкость и газ еще разъединены (см. диаграммы для аргона в [6], [7]). В этом случае мы, оставаясь на критической изотерме, получим уменьшенную фрактальную размерность. Именно так и происходит с экспериментальной кривой (см. рис. 3). Поэтому вплоть до точки $V / R=0.5$ можно считать, что фрактальная размерность меняется от 2.8 к 2.4. Тогда будет полное совпадение с экспериментом.

Однако теоретически мы будем считать размерность равной 2.8 на всем интервале

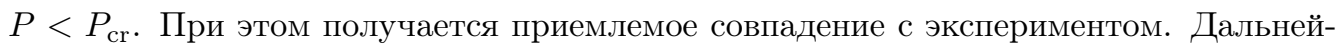
ший учет поправочных членов с помощью $T$-отображения (см. ниже), возможно, даст совпадение с экспериментальной кривой.

Кроме того, прямая $Z=1$ достигается при химическом потенциале, равном $-\infty$. Это означает, что и полная энергия, и число частиц стремятся к нулю. В задаче рассеяния мы рассматривали бесконечный объем. Слишком много нулей и бесконечностей требует привлечения нестандартного анализа. Вместо этого мы рассмотрим в качестве предельной прямую $Z=0.95$. Тогда размерность 2.8 подходит лучше всего. На многих диаграммах исключается окрестность точки $P=0$, поэтому несмотря на расхождение на графиках именно с этой экспериментальной кривой фрактальная размерность 2.8 достаточно точно описывает картину. Это значение получается из задачи рассеяния. Поэтому, указав на возможное расхождение с экспериментом, мы будем рассматривать все точки $Z \leqslant 0.95$, а размерность 2.8 - как фиксированную постоянную размерность. В точке $Z=0.95$ мы можем переходить к классической ван-дер-ваальсовской картине, а поправки будут меньше второго члена вириального разложения [8].

На рис. 4 приводится парастатистическое распределение для классического газа при $T=T_{\text {cr. }}$. Таким образом, вместо перехода в конденсат типа бозе-конденсата мы получаем закон перераспределения энергий. Это совпадает с математически точной асимптотикой при $h \rightarrow 0$ (см. [9]) с учетом соотношения всех малых и больших параметров.

Это также соответствует и методу исследования кластеров и мономеров во флюидах, так как экспериментатор считает процент димеров (критерий Кало - 7\%), т.е. число мономеров и димеров. Это означает, что экспериментатор не различает между собой мономеры и не различает между собой димеры. А это своего рода неразличимость частиц. Димеры создают для мономеров фрактальную размерность. Как она меняется при $P<P_{\text {cr }}$, установить трудно из-за неустойчивости в этой области. На рис. 4 приводится зависимость $Z(P)$ для указанных размерностей.

Теперь определим угол к критической изотерме $T=1$, под которым должна выходить масштабированная (см. выше) изохора.

Вычислим $\left.\frac{d V}{d Z}\right|_{T=1}$ на изотерме. Имеем

$$
Z=\frac{E_{\min }}{E_{\max }}\left(E_{\min }\right),
$$

отсюда

$$
\left.\frac{d Z\left(E_{\min }\right)}{d E_{\min }}\right|_{E_{\max }=E_{\max }^{c r}}=a=1.951
$$


С другой стороны, $Z=P V / R T$ и при $T=1$

$$
\frac{d Z}{d E_{\min }}=\frac{d Z}{d(P V)}
$$

Значит,

$$
\begin{gathered}
\frac{1}{a}=\frac{d P V}{d Z}=V \frac{d P}{d Z}+P \frac{d V}{d Z} \\
\left.\frac{d V}{d Z}\right|_{T=1}=\frac{1}{a P}-\frac{V}{P}\left(\frac{d Z(P)}{d P}\right)^{-1}=\frac{1}{a P}-\frac{Z}{P^{2}} \cdot \frac{1}{(d Z(P)) /(d P)}= \\
=\frac{1}{P}\left(\frac{1}{a}-\frac{Z}{P(d Z / d P)}\right)=\frac{1}{P}\left(\frac{1}{a}-\left(\frac{d \ln Z(P)}{d \ln P}\right)^{-1}\right) .
\end{gathered}
$$

Зависимость $Z(P)$ найдена из соотношения

$$
\begin{gathered}
d \mu=V d P=\frac{d P Z(\mu)}{P}, \\
\frac{d \mu}{d P}=\frac{Z(\mu)}{P},\left.\quad \mu(P)\right|_{P=1}=0 .
\end{gathered}
$$

Отсюда получаем зависимости $\mu(P)$ и $Z(\mu(P))$ (см. рис. 4).

Для идеального газа (см. [1]) при $\infty \geqslant \mu>-\infty$

$$
Z(\mu)=\frac{\Gamma(\gamma)}{\Gamma(\gamma+1)} \frac{\int \varepsilon^{\gamma+1}\left(\frac{1}{e^{\varepsilon-\mu}-1}-\frac{N_{\mathrm{cr}}}{e^{N_{\mathrm{cr}}(\varepsilon-\mu)}-1}\right) d \varepsilon}{\int \varepsilon^{\gamma}\left(\frac{1}{e^{\varepsilon-\mu}-1}-\frac{N_{\mathrm{cr}}}{e^{N_{\mathrm{cr}}(\varepsilon-\mu)}-1}\right) d \varepsilon} .
$$

При $\mu \leqslant 0$ в случае $\gamma>2$ вторым членом в числителе и знаменателе можно пренебречь(см. рис. 4). $Z(\mu(P))$ при $P>1, \mu>0$ дает хорошее приближение для $Z(P)$, $T=1, \gamma=0.2$ (размерность 2.4). При малом положительном $\mu$ особенности числителя и знаменателя сокращаются, $Z(\mu)$ вначале немного убывает, а затем при $\mu=0.1$ и $N_{\mathrm{cr}} \rightarrow \infty Z(\mu)=\mu$. Тогда $Z(\mu(P))$ при $P>1.5$ переходит в прямую линию.

\section{2. ПЕРЕХОД В КОНДЕНСАТ В ДВУМЕРНОМ СЛУЧАЕ ДЛЯ ЛЮБОГО КЛАССИЧЕСКОГО ГАЗА}

Повторим некоторые утверждения предыдущей работы в несколько более общем виде, приспособленном для определения двумерного конденсата.

Мы будем говорить о дискретном фазовом пространстве и о временных рядах. Это не связано с константой Планка, а связано с точностью компьютера и разрешающей способностью измерительных приборов ${ }^{3)}$. Таким образом, мы изначально задаем "клетки" фазового объема (см. [10]), откуда получаем число собственных значений $s\left\{\varepsilon_{1}, \varepsilon_{2}, \ldots, \varepsilon_{s}\right\}$. Далее задаем $s$ и лишь затем "число частиц" $N(s) \rightarrow \infty$

\footnotetext{
3) Именно эти соображения в конкретных случаях позволяют представить в безразмерном виде как "клетки” фазового объема, так и уровни энергии.
} 
при $s \rightarrow \infty$. Тем самым с точностью до унитарного преобразования (представления) можно сказать, что задана $s$-мерная самосопряженная матрица или самосопряженный оператор. А так как мы изначально рассматриваем задачу о частицах, то возникает дуализм волна-частица в конечномерном ( $s$-мерном) гильбертовом пространстве $H$, отвечающем одной частице.

Для $N$ частиц мы рассмотрим тензорное произведение $N$ одночастичных гильбертовых пространств и соответствующий $N$-частичный уровень энергии $\mathcal{E}$ (глобальная энергия). При этом мы будем рассматривать лишь симметричные по перестановке частиц собственные функции. Глобальная энергия $\mathcal{E}$ имеет вид

$$
\mathcal{E}=\sum_{i=1}^{s} N_{i} \varepsilon_{i}, \quad \sum_{i=1}^{s} N_{i}=N .
$$

Кратность этого уровня $\mathcal{E}$ равна числу всех возможных вариантов такого представления (см. [11]).

ОПРЕДЕЛЕНИЕ 1. Будем говорить, что набор $\left\{\varepsilon_{i}\right\}$ находится в общем положении, если все кратности, отвечающие собственному значению $\mathcal{E}$ (глобальной энергии), равновероятны.

ОПРЕДЕЛЕНИЕ 2. Плотностью $\rho$ и объемом $V$ числа частиц $N$ относительно числа $s$ (точности компьютера) мы будем называть величины

$$
\rho=\frac{(\mathcal{E} / N)^{3 / 2} N}{6 \pi^{2} s}, \quad V=\frac{N}{\rho} .
$$

Мы предполагаем, что $\rho \rightarrow$ const при $s \rightarrow \infty$.

Приведем общую теорему, отвечающую заданной функции $H(p, q) \geqslant 0, p \in \mathbb{R}^{l}$, $q \in \mathbb{R}^{l}, 0<l \leqslant 3$.

Предположим, что функция $H(p, q)$ медленно меняется по переменной $q$, и положим $H(p, q)=E(p, \varepsilon q), \varepsilon=1 / \sqrt[3]{V}, E(p, x) \equiv 0$ при $x \neq \Omega$, где $\Omega$ - некоторый объем единичного размера. Предположим также, что функция $E(p, x)$ гладкая.

Пусть $\mathcal{E}$ и $s \gg 1$ - заданные числа, $\varepsilon_{1} \ll \mathcal{E}$,

$$
\varepsilon_{i}=\iint_{\varepsilon_{1}(i+1) \leqslant H(p, q) \leqslant \varepsilon_{1} i} H(p, q) d p d q, \quad i=2,3, \ldots .
$$

Рассмотрим соотношение

$$
\sum_{i=0}^{s} \varepsilon_{i} N_{i}=\mathcal{E}, \quad \varepsilon_{0}=0,
$$

где $N_{i}$ - целые числа такие, что $\sum_{0}^{s} N_{i}=N$. Предположим, что набор $\left\{\varepsilon_{i}\right\}_{i=1}^{s}$ находится в общем положении, а $N \rightarrow \infty$ таково, что плотность $\rho$ при $s \rightarrow \infty$ стремится к константе.

Существуют $\beta \rightarrow 0$ и $N_{\mathrm{cr}} \rightarrow \infty$, удовлетворяющие соотношениям

$$
\begin{gathered}
\int_{0}^{\infty} H(p, q)\left\{\frac{1}{e^{\beta H(p, q)}-1}-\frac{N_{\mathrm{cr}}}{e^{\beta H(p, q) N_{\mathrm{cr}}}-1}\right\} d p d q=\mathcal{E}, \\
\int_{0}^{\infty}\left\{\frac{1}{e^{\beta H(p, q)}-1}-\frac{N_{\mathrm{cr}}}{e^{\beta H(p, q) N_{\mathrm{cr}}}-1}\right\} d p d q=N_{\mathrm{cr}} .
\end{gathered}
$$


Рассмотрим уравнения для определения констант $b$ и $\kappa$ :

$$
\begin{gathered}
\int_{0}^{\infty} H(p, q)\left\{\frac{1}{e^{b(H(p, q)+\kappa)}-1}-\frac{N}{e^{b N(H(p, q)+\kappa)}-1}\right\} d p d q=\mathcal{E}, \\
\int_{0}^{\infty}\left\{\frac{1}{e^{b(H(p, q)+\kappa)}-1}-\frac{N}{e^{b N(H(p, q)+\kappa)}-1}\right\} d p d q=N .
\end{gathered}
$$

Теорема 1. А. Если $N \leqslant N_{\mathrm{cr}}$, то решение уравнений (7) существует и единственно при $\kappa \geqslant 0, b \geqslant 0$. При $N=N_{\text {сг }}$ получаем $\kappa=0, b=\beta, N_{0}=0$.

Б. Если $\varphi(\xi)$ - любая ограниченная кусочно-гладкая функиия (с конечным числом разрывов), непрерывная в точках bi, то $N_{0}=0$, а разность

$$
\sum_{i=1}^{\infty} N_{i} \varphi(b i)-\int_{0}^{\infty} \varphi(H(p, q))\left\{\frac{1}{e^{b(H(p, q)+\kappa)}-1}-\frac{N}{e^{b N(H(p, q)+\kappa)}-1}\right\} d p d q
$$

деленная на $N$, стремится по вероятности $к$ нулю при $N \rightarrow \infty$.

В. Пусть $N>N_{\mathrm{cr}}$. Тогда существует такое $\delta>0$, что

$$
\mathbf{P}\left\{\left|N_{0}-\left[N-N_{\mathrm{cr}}\right]\right| \geqslant \delta_{1} N_{\mathrm{cr}}\right\} \leqslant e^{-\left|N-N_{\mathrm{cr}}\right|^{\delta}},
$$

где $\delta_{1}$ - сколь угодно малое, не зависящее от $N_{\mathrm{cr}}$ число, а $\mathbf{P}$ есть отношение числа вариантов, удовлетворяющих условию в фигурных скобках, к общему числу вариантов при $N=N_{\mathrm{cr}}$.

Элементы последовательности $N_{1}, N_{2}, \ldots, N_{i}, i \leqslant A / \beta$, где $A$ - сколь угодно большое число, не зависящее от $\beta$, удовлетворяют соотношению

$$
\begin{gathered}
\frac{1}{N_{\mathrm{cr}}}\left[\sum_{i=1}^{[A / \beta]} N_{i} \varphi(\beta i)-\int_{0}^{\infty} \varphi(H(p, q))\left\{\frac{1}{e^{\beta H(p, q)}-1}-\frac{N_{\mathrm{cr}}}{e^{\beta N_{\mathrm{cr}} H(p, q)}-1}\right\} d p d q\right] \stackrel{\mathrm{P}}{\rightarrow} 0 \\
n p u \quad N_{\mathrm{cr}} \rightarrow \infty,
\end{gathered}
$$

где $\beta$ определяется из соотношения (5).

СЛЕДСТвИЕ 1. В случае, когда $l=2$, a $H(p, q)=p^{2} /(2 m)$ не зависит от $q$, конденсат достигается при $\kappa=0$ и при $N_{\mathrm{cr}}$, удовлетворяющем соотношению $N_{\mathrm{cr}}=$ const $\sqrt{E} \ln N_{\mathrm{cr}}$. Поскольку $\ln N \approx 28$, условие $\rho=$ const выполняется [12], [13].

Возвратимся к спору Больцмана с математиками (см. [1]) с точки зрения приведенной теоремы. Математики полагали, что молекулы газа “помнят" о своих начальных условиях и что процесс перехода от классической динамики к газодинамике детерминирован. А молекулы “забыли” о своем происхождении (точнее, компьютер не удержал в памяти начальные данные). Уменьшим их исходные начальные скорости, а с ними и средние скорости (скорости, отвечающие средней энтропии $\mathcal{E} / N$ ) в некоторое число раз. Тогда время их перехода в состояние, когда память компьютера не могла сохранить их начальные состояния (переход в индетерминизм, в хаос), вырастет в это же число раз. Однако в силу теоремы 1 оказывается, что если среднюю скорость уменьшить ниже критического числа $\mathcal{E} / N_{\mathrm{cr}}$, то компьютер 
"вспомнит" происхождение части молекул, и совершится фазовый переход из индетерминированного состояния в частично детерминированное, что соответствует двухжидкостной модели Тисса-Ландау. Если $N \ll N_{\mathrm{cr}}$, то распределение (8) переходит в распределение Максвелла-Больцмана.

Вспомним фокус Коровьева-Фагота, описанный в романе М. А. Булгакова "Мастер и Маргарита", о котором говорилось в наших предыдущих работах [3], [14]. В варьете над зрителями были рассыпаны купюры достоинством в один червонец. Считаем, что все варианты разложения $n$ купюр по $k$ зрителям равновозможны (условие хаотичности). Рассмотрим два примера, касающиеся фокуса Фагота.

Пример 1. Пусть $n$ купюр распределено по $k_{0}$ зрителям, где число $k_{0}$ такое, что

$$
p_{k_{0}}(n)=\sup _{k} p_{k}(n) .
$$

(Ясно, что при $k=1$ и при $k=n$ число вариантов равно единице, а значит, существует такой супремум.) Тогда $k_{0}$ зрителей с большой вероятностью получат некоторое количество купюр. Общее число вариантов распределения купюр по зрителям равно $p_{k}(n)$.

Теперь предположим, что число зрителей $2 k_{0}$. Тогда, грубо говоря, $k_{0}$ (т.е. половина) зрителей получат по сколько-то купюр, а $k_{0}$ останутся ни с чем. В силу того что $k_{0}$ зрителей получили ноль купюр, число вариантов распределения купюр по зрителям также равно $p_{k_{0}}(n)$.

Пример 2. Объединим зрителей в пары с условием, что если кто-то из пары не получит ни одной купюры, то та сумма, которую получил другой, делится, например, пополам. Так как теперь образовалось $k_{0}$ пар, то число всех вариантов будет равно числу вариантов в первом примере.

Значит, колмогоровская сложность (энтропия Хартли) в обоих примерах одна и та же и равна $\log _{2} p_{k_{0}}(n)$, где $k_{0}$ - число пар. Следовательно, оба случая равновероятны, т.е. если зрителей было $2 k_{0}$, то $k_{0}$ из них ничего не получили, и если зрители объединились в пары, то все пары получили купюры, но число вариантов распределения все равно осталось равным $p_{k_{0}}(n)$, и предпочесть один из примеров мы не можем. Эти две реализации равновозможны. Иначе говоря, может быть, зрители объединились в пары, а может быть, $k_{0}$ зрителей остались без купюр. Если говорить о социально-экономической выгодности, лучше бы зрители объединились в пары.

ЗАмЕчАниЕ 2. Если число зрителей равно $k$ и выполняется условие $k_{0}<k<2 k_{0}$, то нетрудно найти такое соотношение пар (димеров) и одиночек (мономеров) с учетом всех возможных комбинаций $l$ пар и $m$ одиночек, $k=m=2 l$, при котором число вариантов разложения числа будет равно $p_{k_{0}}(n)$. Для частиц это соответствует газу, состоящему из смеси двух типов тождественных частиц - мономеров и димеров, при этом общая энергия постоянна, а число вариантов максимально.

Таким образом, априори неизвестно, что возникнет при фазовом переходе идеального бозе-газа в конденсат: пары или, как ранее предполагалось в физике, частицы на нижнем уровне. Если рассматривать отталкивающий потенциал, как это 
делал Боголюбов в своей знаменитой работе [15], то этот вопрос остается нерешенным. Но если рассматривать потенциал Леннарда-Джонса, то, как показал автор в работе [6], возникают пары. При этом существенно использовалась теорема Андронова-Понтрягина-Витта [16], в силу которой время пребывания частицы внутри барьера, имеющего место для потенциала Леннарда-Джонса, зависит от уровня шума или температуры газа.

ЗАМЕЧАНИЕ 3 (о ВОЗНИКНОВЕНИИ ДВУМЕРНОГО КОНДЕНСАТА). Как уже неоднократно отмечал автор, двумерный конденсат возникает для теплопроводности в двумерном случае, т.е., например, для вращающихся димеров в точке, в которой химический потенциал $\mu$ обращается в нуль. Но этот факт противоречит понятию "термодинамического предела", т.е. случаю, когда число частиц $N \rightarrow \infty$ и объем $V \rightarrow \infty$, так что $N / V=$ const. Мы говорим здесь о термодинамике, но о понятии термодинамического предела пора забыть по той причине, что оно запутывает современную науку, где проявляются и другие малые параметры, не говоря уж о постоянной Планка $\hbar$. Эксперименты можно проводить на спутниках, где отсутствуют земное притяжение и атмосферное давление. В этом смысле я понимаю тех великих физиков (к ним относится, в частности, Зельдович), которые не признавали асимптотических разложений, а прекрасно знали цифры (и даже держали их в голове).

Если $N \rightarrow \infty$, то $\ln N \rightarrow \infty$, а логарифм числа Авогадро равен примерно 28, т.е. не так велик. При давлении примерно в 20 раз меньше атмосферного газ будет столь разрежен, что число 28 не будет велико, а значит, плотность $N / V$ не будет стремиться к бесконечности.

Как уже отмечалось в работе [1], поправку к $T_{\text {сr }}$ для каждого отдельного газа, приведенного в таблице, можно получить, построив $T$-отображение (см. следующий раздел) с помощью соотношения

$$
W(q)=-P^{\Delta} V+\frac{\iint d p^{\prime} d q^{\prime} W\left(\left|q-q^{\prime}\right|\right) u\left(p^{2} /(2 m)+W\left(q^{\prime}\right) t\right)}{\iint d p^{\prime} d q^{\prime} u\left(p^{2} /(2 m)+W\left(q^{\prime}\right)\right)},
$$

где функция $u(E)$ выбирается в виде $u(E)=\left(e^{(E-\mu) / \theta}-1\right)^{-1}, W\left(\left|q-q^{\prime}\right|\right)$ - эффективный потенциал Леннарда-Джонса:

$$
\Phi=4 \varepsilon\left(\frac{a^{12}}{r^{12}}-\frac{a^{6}}{r^{6}}\right), \quad r=\left|q-q^{\prime}\right| .
$$

Уравнение (9) есть уравнение самосогласованного поля. Построение $T$-отображения [17] для него приводится в следующем разделе. Для того чтобы уточнить предложенную схему и учесть детерминистическое давление, отметим следующее обстоятельство.

Известно, что если две рассеивающиеся друг на друге частицы вместе движутся равномерно, то можно отделить совместное движение от рассеяния, выделив в гамильтониане член $H_{2}=\left(p_{1}+p_{2}\right)^{2} / m, H=H_{1}+H_{2}$, для центра масс. Это же можно сделать и для многих частиц. Более того, если каждая частица притягивается к земле, то вычитая из гамильтониана потенциал $m g z_{i}$, где $z$ - компонента, направленная к земле, для каждой частицы можно отделить не зависящий от взаимодействия между частицами гамильтониан $H_{2}$, отвечающий ускоренному падению 
каждой частицы, так что скобка Пуассона между $H_{1}$ и оставшимся гамильтонианом $H_{2}$, описывающая взаимодействие, будет равна нулю. Таким образом, потенциал, описывающий задачу рассеяния, в результате общего ускорения движения не изменится.

Если общее потенциальное поле, действующее на каждую частицу, линейно по координате $q$, то можно выделить задачу рассеяния, не зависящую от этого внешнего поля. Выделенный потенциал в двухчастичном случае имеет вид

$$
H_{2}=\frac{\left(p_{1}+p_{2}\right)^{2}}{m}+\alpha\left(q_{1}+q_{2}\right) .
$$

Если же внешнее поле квадратично по $|q|$, то учитывая, что

$$
\alpha_{1} q_{1}^{2}+\alpha_{1} q_{2}^{2}=\frac{\alpha_{1}}{2}\left\{\left|q_{1}-q_{2}\right|^{2}+\left|q_{1}+q_{2}\right|^{2}\right\},
$$

где $\alpha_{1}, \alpha_{2}$ - константы, мы можем добавить член $\left(\alpha_{1} / 2\right)\left|q_{1}-q_{2}\right|^{2}$ к гамильтониану $H_{1}$, т.е., изменив взаимодействие, мы снова сможем разделить общий гамильтониан на гамильтониан $H_{1}$, отвечающий задаче рассеяния, и гамильтониан

$$
H_{2}=\frac{\left(p_{1}+p_{2}\right)^{2}}{m}+\alpha\left(q_{1}+q_{2}\right)+\frac{\alpha_{1}}{2}\left(q_{1}+q_{2}\right)^{2} .
$$

Однако в последнем к потенциалу взаимодействия добавляется слагаемое $\left(\alpha_{1} / 2\right) \mid q_{1}-$ $\left.q_{2}\right|^{2}$, что, в отличие от предыдущих примеров, изменит потенциал взаимодействия.

В равновесной термодинамике воздействие внешнего детерминированного давления должно быть очень небольшим, чтобы затем выравнять температуру, вновь привести к равновесию, затем снова надавить слегка поршнем и т.д. При этом в силу того, что процесс должен быть медленным, добавку к средней энергии можно считать с точностью до кубических членов. Это немного изменит потенциал взаимодействия в стационарной задаче рассеяния. Затем после установления равновесия в системе можно снова слегка увеличить давление поршнем и такими шажками прийти к окончательному равновесному распределению.

Такой процесс по аналогии с $T$-произведением автор назвал $T$-отображением. Только последовательно применив указанный процесс, можно добиться полного совпадения с экспериментом.

Например, при некоторых условиях при изотермическом процессе, как известно, происходит изменение свободной энергии

$$
\Delta E=\frac{P_{0}}{\rho_{0}} \ln \left(1+\frac{\Delta \rho}{\rho_{0}}\right),
$$

где $\rho_{0}$ - плотность, $\Delta \rho$ - добавленная плотность за счет увеличения давления поршнем (см. [18]). Для жидкого флюида это соотношение неизвестно, однако известна адиабата

$$
\frac{P}{P_{0}}=\left(\frac{\rho}{\rho_{0}}\right)^{11 / 6} .
$$

По существу, после перераспределения энергий от значения 0.444 к значению 0.296 мы приходим к некоторому "жидкому" флюиду для всех газов, для которых экспериментальный график справедлив. Следовательно, далее для описания неравновесного процесса мы можем использовать гидродинамику, для которой несущественно, 
какого состава жидкость рассматривается (важно только, что она не ньютоновская). А из этого следует, что закон соответственных состояний выполняется сразу для всех газов, приводимых в таблице выше.

Поскольку объем $V$ можно представить в виде цилиндра, в котором $z$ - координата вдоль цилиндра, а поршень, увеличивающий давление, имеет своим основанием крышку площадью $\varsigma$, то $\Delta P \varsigma$ - это сила, а $\Delta P \varsigma \Delta z$ - потенциальная энергия. Мы учитываем столь малую величину $\Delta P$, чтобы сопротивление газа при увеличении давления на $\Delta P$, дающем уменьшение объема $\Delta V=\varsigma \Delta z$, было ограничено величиной $O\left((\Delta z)^{3}\right)$.

После перехода данного неравновесного процесса в равновесный это, как отмечалось выше, несколько изменит потенциал рассеяния на величину $\alpha\left(z_{1}-z_{2}\right)^{2} \Theta\left(\mid z_{1}-\right.$ $\left.z_{2} \mid-\varepsilon\right)$, где $\Theta=0$ при $z_{1}-z_{2}>\varepsilon$ и $\Theta=1$ при $z_{1}-z_{2}<\varepsilon$, а $\varepsilon$ достаточно мало. Процесс $T$-отображения [17] для изотермы при увеличении давления идет следующим образом: по адиабате объем уменьшается, по измененной (в силу добавки к потенциалу Леннарда-Джонса) изохоре температура опускается до $T=1$. Нужно найти изотерму с учетом соотношения для $d V / d Z$ на изотерме (см. выше), определяющего угол между изохорой и изотермой. Повторяя эту процедуру с установлением равновесия, мы получим зависимость $T_{\text {cr }}$ от давления. Для получения более точных результатов для конкретного газа (из рассматриваемых в таблице) нужно использовать соотношение (9).

Это, разумеется, касается гладкой части приведенных экспериментальных кривых. Наше нулевое приближение для негладкой части изотермы $T=1$ дало абсолютно точное значение, поэтому указанный процесс в конечном счете должен дать точное совпадение.

ЗАМЕчАНИЕ 4. При вычислении поправок и применении $T$-отображения нужно помнить, что температура $T$ выступает как бы в трех ипостасях. Во-первых, она определяет энергию взаимодействующих между собой частиц $E=T / 4$.

Во-вторых, отношение энергии $E_{\min } / E_{\max }\left(E_{\min }\right)$ есть отношение энергии к полной энергии мономеров как функция от энергии мономеров, т.е. $P V / T$. А в силу того, что объем $V$ есть объем мономеров, как обычно исчисляемый в $\mathrm{cm}^{3} /$ моль, т.е. объем, деленный на газовую постоянную $R$, то $E_{\min } / E_{\max }\left(E_{\min }\right)$ есть фактор сжимаемости $Z$. Он вычисляется как функция от $E_{\min }$, т.е. от энергии мономеров, равной $P V$.

В-третьих, температура входит в парастатистическое, бозевское и максвелловское распределения. Задача рассеяния дает нам изохору. При увеличении температуры и давления немного меняется потенциал взаимодействия, а значит, меняется и отвечающая задаче рассеяния изохора, которую нужно рассматривать лишь от такой точки $E$, которая отвечает данной температуре. Ниже этой точки $E$ изохора в силу приведенной концепции может быть искажена. При повышении давления изохора вытягивается, т.е. последовательные приближения изменяют ямки и растягивают изохору при приближении к температуре Бойля. Таким образом, казалось бы, мы меняем потенциал взаимодействия, но в силу этого замечания мы меняем масштабирование (см. выше). Это естественно, поскольку потенциал Леннарда-Джонса сам 
выступает в двух ролях - он определяет задачу рассеяния и он же обеспечивает сопротивление давлению поршня и определяет одетый потенциал среднего поля. Эта "самосогласованность" потенциала парного взаимодействия и проявляется в растяжении изохоры при увеличении давления. Процесс $T$-отображения отражает это самовоздействие парного взаимодействия.

По существу, нулевое приближение уже дает достаточно точную картину. Метод $T$-отображения может уточнить картину для каждого отдельного газа, поскольку в парастатистическое распределение входят индивидуальные параметры данного газа.

\section{3. Т-ОТОБРАЖЕНИЕ ДЛЯ ЗАДАЧ СРЕДНЕГО ПОЛЯ}

3.1. T-отображения и метод ломаных Эйлера. Самосогласованные уравнения для плазмы без учета столкновений описывают взаимодействие частицы с усредненными полями других частиц. Эти нелинейные уравнения обычно содержат производные первого порядка, а их коэффициенты зависят от свертки неизвестной функции $u(x, t)$ с некоторыми заданными потенциалами. Из физических соображений следует, что коэффициенты зависят от значения неизвестной функции в некоторый предшествующий момент $u(x, t-\delta)$ (эффект запаздывания или релаксации). Однако параметр $\delta$ настолько мал, что в уравнении мы им пренебрегаем. Но при составлении разностной схемы для таких уравнений этот факт, по существу, учитывается, поскольку значения коэффициентов берутся на предыдущем шаге по сравнению с решением.

Мы введем специальное понятие $T$-отображения, в котором на самом деле отражается факт запаздывания коэффициентов уравнения и которое дает метод построения решения нелинейного уравнения шагами по $t$. В этом смысле $T$-отображение иногда стоит ближе к реальным физическим процессам, чем само уравнение. С другой стороны, как будет показано ниже, метод $T$-отображений обобщает метод ломаных Эйлера. Таким образом, метод $T$-отображений может служить конструктивным методом доказательства существования решений. Кроме того, $T$-отображения тесно связаны с понятием нелинейного континуального интеграла, и, с нашей точки зрения, решения характеристических уравнений для континуального интеграла естественно записывать в виде T-отображения, поскольку континуальный интеграл может быть также интерпретирован как метод построения решения шагами по $t$.

$T$-отображения, ассоциированные с нелинейными уравнениями квантовой механики, являются естественным обобщением $T$-произведений, причем в этом случае формулы "выпутывания" Фейнмана переносятся на T-отображения. Именно это фундаментальное обстоятельство позволяет построить квазиклассическую асимптотику нелинейных уравнений квантовой механики и нелинейный континуальный интеграл, отвечающий им.

Уравнение Власова играет в наших построениях такую же роль, как уравнение Лиувилля классической механики для фейнмановского континуального интеграла.

В этом разделе после изложения общих свойств $T$-отображений мы рассмотрим нелинейные уравнения, обобщающие уравнения Власова.

Дадим сначала определение T-отображения и изучим его связь с методом ломаных Эйлера. Определение $T$-отображения мы дадим в три этапа. Прежде чем 
сформулировать общее определение, мы рассмотрим линейный случай и затем случай псевдодифференциальных операторов.

Многие уравнения математической физики, в частности квантовой механики, являются эволюционными уравнениями вида

$$
-i \frac{\partial \psi(t)}{\partial t}+\widehat{H}(t) \psi(t)=0
$$

где $\psi(t)$ - функция со значениями в гильбертовом пространстве, $\widehat{H}(t)$ - заданный линейный неограниченный самосопряженный оператор в этом же пространстве. Формально решение такого уравнения, следуя Фейнману, записывают в виде $T$-произведения следующим образом:

$$
\psi(t)=\exp \left\{-i \int_{t_{0}}^{t} \widehat{H}_{\tau} d \tau\right\} \psi\left(t_{0}\right) .
$$

Под этим понимается следующий предел “хронологического" произведения:

$$
\psi(t)=\lim _{\delta_{N} \rightarrow 0} e^{-i \Delta t_{N-1} \widehat{H}\left(t_{N-1}\right)} \ldots e^{-i \Delta t_{0} \widehat{H}\left(t_{0}\right)} \psi\left(t_{0}\right),
$$

где $t_{0}<t_{1}<\cdots<t_{N} \equiv t$ - некоторое разбиение отрезка $\left[t_{0}, t\right]$ на $N$ интервалов длины $\Delta t_{j}=t_{j+1}-t_{j}$, а $\delta_{N}=\max \lim _{j} \Delta t_{j}$.

Таким образом, для получения функции $\psi$ в момент времени $t+d t$ нужно применить инфинитезимальный оператор $e^{-i d t \widehat{H}(t)}$ к функции $\psi$ в момент времени $t$ :

$$
\psi(t+d t)=e^{-i d t \widehat{H}(t)} \psi(t) .
$$

Для предела (13) мы введем следующее обозначение:

$$
\psi(t)=\left[\prod_{\tau=t_{0}}^{t} e^{-i d \tau \stackrel{\tau}{\hat{H}}(\tau)}\right] \psi\left(t_{0}\right) .
$$

Аналогично определяется нелинейное T-отображение. Вначале дадим определение в том частном случае, который в дальнейшем будет играть важную роль. Пусть $B_{n}$ - некоторое банахово пространство функций от $n$ вещественных переменных и $H: B_{n} \rightarrow B_{2 n}-$ некоторое нелинейное отображение. Для каждой функции $\psi \in B_{n}$ ее образ $H[\psi]$ есть функция от $2 n$ переменных. Обозначим эти переменные через $x=\left(x_{1}, \ldots, x_{n}\right), p=\left(p_{1}, \ldots, p_{n}\right)$. Таким образом, $H[\psi](x, p)$ есть функция на $\mathbb{R}^{2 n}$. Сопоставим ей псевдодифференциальный оператор $\widehat{H}[\psi] \stackrel{\text { def }}{=} H[\psi](\stackrel{2}{x}, \stackrel{1}{p})$, где $p=-i h / \partial_{1} x, h-$ некоторый вещественный параметр (постоянная Планка), номера 1 и 2 обозначают порядок действия оператора.

Рассмотрим эволюционное уравнение

$$
i \frac{\partial \psi}{\partial t}=(\widehat{H}[\psi]) \psi
$$

Решение этого уравнения будем формально представлять в виде

$$
\psi(t)=\lim _{\delta_{N} \rightarrow 0} \psi_{N},
$$


где $\psi_{N}$ определяется из следующей рекуррентной системы равенств:

$$
\begin{aligned}
\psi_{k+1} & =e^{-i \Delta t_{k} \widehat{H}\left[\psi_{k}\right]} \psi_{k}, \quad k=0,1, \ldots, N-1, \\
\psi_{0} & =\psi\left(t_{0}\right),
\end{aligned}
$$

или, в дифференциальной форме,

$$
\psi(t+d t)=e^{-i d t \widehat{H}[\psi(t)]} \psi(t) .
$$

Такое представление функции $\psi(t)$ мы будем записывать в виде

$$
\psi(t)=\left[\prod_{\tau=t_{0}}^{t} e^{-i d \tau \stackrel{\tau}{\hat{H}}[\psi(t)]}\right] \psi\left(t_{0}\right) .
$$

Таким образом, значение функции $\psi$ в момент $t+d t$ может быть получено применением инфинитезимального оператора $e^{-i d t \widehat{H}[\psi(t)]}$ к функции $\psi(t)$.

Отображение $\psi\left(t_{0}\right) \rightarrow \psi(t)$, построенное по формулам (15), (16), мы назовем T-отображением по аналогии с термином "T-произведение", которым обычно обозначают мультипликативный интеграл (12). Таким образом, T-произведение есть частный случай $T$-отображения, когда оператор $\widehat{H}$ линеен.

Дадим теперь самое общее определение $T$-отображения, не связанное ни с псевдодифференциальными операторами, ни с каким-либо эволюционным уравнением.

Рассмотрим некоторое банахово пространство $B$ и обозначим через $C([0, t], B)$ банахово пространство непрерывных функций $\psi=\psi(t)$ на $[0, t] \subset \mathbb{R}$ со значениями в $B$, наделенное нормой

$$
\|\psi\|_{C([0, t], B)}=\sup \lim _{0 \leqslant \tau \leqslant t}\|\psi(\tau)\|_{B}
$$

Пространство $C([0,0], B)$ отождествим с $B$.

Пусть $t \geqslant t^{\prime}$. Каждой функции $\psi \in C([0, t], B)$ сопоставим ее сужение на $\left[0, t^{\prime}\right]$, получим новую функцию $\psi^{\prime} \in C\left(\left[0, t^{\prime}\right], B\right)$. В дальнейшем мы будем опускать знак штриха и обозначать через $\psi$ как саму исходную функцию, так и все ее сужения на меньшие отрезки.

Пусть заданы $\varepsilon_{0}>0, T_{0}>0$ и при любых $t, \varepsilon$ таких, что $0 \leqslant t \leqslant T_{0}$, $0 \leqslant \varepsilon \leqslant \varepsilon_{0}$, определено некоторое двупараметрическое семейство отображений $W_{t, \varepsilon}: C([0, t], B) \rightarrow B$ такое, что при любом $t \in\left[0, T_{0}\right]$ и любом $v \in C([0, t], B)$ элемент $W_{t, \varepsilon}[v]$ непрерывно зависит от $\varepsilon$ и $W_{t, 0}[v]=v(t)$. Положим

$$
\left(S_{t, \varepsilon}[v]\right)(\tau)= \begin{cases}W_{t, \tau-t}[v], & t \leqslant \tau \leqslant t+\varepsilon \\ v(\tau), & \tau \leqslant t\end{cases}
$$

Получим отображение $S_{t, \varepsilon}: C([0, t], B) \rightarrow C([0, t+\varepsilon], B)$. Обозначим через $0 \equiv$ $t_{0}<\cdots<t_{N} \equiv T_{0}$ точки разбиения отрезка $\left[0, T_{0}\right]$ на интервалы $\Delta t_{j}=t_{j+1}-t_{j}$, где $j=0, \ldots, N$. Максимальную длину этих интервалов обозначим $\delta_{N}=\max \lim _{j} \Delta t_{j}$.

Пусть $v_{0} \in B$. Положим

$$
v_{N}=S_{t_{N-1}, \Delta t_{N-1}} \circ \cdots \circ S_{t_{0}, \Delta t_{0}}\left[v_{0}\right] .
$$


Здесь знак о обозначает композицию нелинейных отображений.

Вообще говоря, предел $\lim _{\delta_{N} \rightarrow 0, N \rightarrow \infty} v_{n}$ по норме пространства $C\left(\left[0, T_{0}\right], B\right)$ существует не при всех $v_{0} \in B$. Пусть $D-$ подмножество в $B$, на котором этот предел существует.

ОПРЕДЕЛЕНИЕ 3. Пусть $v_{0} \in D$ и $v=\lim _{\delta_{N} \rightarrow 0} v_{N}$. Отображение $v_{0} \rightarrow v$ назовем $T$-отображением с образующей $S_{t, \varepsilon}$ и обозначим

$$
v(t)=\left(\prod_{\tau=0}^{t} \circ \stackrel{\tau}{\tau}_{\tau, d \tau}\right)\left[v_{0}\right]
$$

Будем говорить, что $T$-отображение с образующей $S_{t, \varepsilon}$ существует на элементе $v_{0}$ на отрезке времени $\left[0, T_{0}\right]$ в норме пространства $C\left(\left[0, T_{0}\right], B\right)$.

Пример 3. Пусть $\tau \in\left[0, T_{0}\right]$ и $R_{\tau}$ - непрерывное отображение $C([0, \tau], B)$ в $B$. Рассмотрим задачу Коши

$$
\begin{aligned}
\frac{d \psi(\tau)}{d \tau} & =R_{\tau}[\psi], & & \psi \in C\left(\left[0, T_{0}\right], B\right), \\
\left.\psi(\tau)\right|_{\tau=t} & =v(t), & & v \in C([0, t], B) .
\end{aligned}
$$

Здесь $t$ - фиксированная точка из отрезка $\left[0, T_{0}-\varepsilon_{0}\right]$. Решение этой задачи Коши $\psi(\tau)$ при каждом $\tau$ есть элемент пространства $B$.

Рассмотрим семейство отображений

$$
W_{t, \varepsilon}: v \rightarrow \psi(t+\varepsilon), \quad \varepsilon \leqslant \varepsilon_{0},
$$

соответствующее семейство $S_{t, \varepsilon}$ действует по формуле

$$
\left(S_{t, \varepsilon}[v]\right)(\tau)= \begin{cases}\psi(\tau), & t \leqslant \tau \leqslant t+\varepsilon \\ v(\tau), & \tau \leqslant t\end{cases}
$$

Очевидно, что элемент $v_{N}=S_{t_{N-1}, \Delta t_{N-1}} \circ \cdots \circ S_{t_{0}, \Delta t_{0}}\left[\psi_{0}\right]$ является решением задачи Коши

$$
\frac{d \psi(\tau)}{d \tau}=R_{\tau}[\psi],\left.\quad \psi\right|_{\tau=0}=\psi_{0} \in B
$$

Следовательно, при любом $N \psi=v_{N}$, т.е.

$$
\psi=S_{t_{N-1}, \Delta t_{N-1}} \circ \cdots \circ S_{t_{0}, \Delta t_{0}}\left[\psi_{0}\right]
$$

При $N \rightarrow \infty, \delta_{N} \rightarrow 0$ получаем $\psi=\lim v_{n}$, и, значит, $S_{t, \varepsilon}$ является образующей $T$-отображения $\psi_{0} \rightarrow \psi$. Ясно, что любой оператор $S_{t, \varepsilon}^{\prime}$ такой, что $S_{t, \varepsilon}-S_{t, \varepsilon}^{\prime}=O\left(\varepsilon^{2}\right)$, будет служить образующей этого же $T$-отображения.

Рассмотрим теперь Т-отображение, ассоциированное с более общим эволюционным уравнением.

Пример 4. Пусть $B_{1} \subset B_{2}$ - два банаховых пространства с нормами $\|\cdot\|_{1} \geqslant$ $\|\cdot\|_{2}$. Обозначим через $C_{j}(t)=C\left([0, t], B_{j}\right)$ пространство непрерывных отображений $[0, t] \rightarrow B_{j}, j=1,2$. Тогда $C_{1}(t) \subset C_{2}(t)$. 
Пусть при каждом $t \in\left[0, T_{0}\right]$ задано непрерывное отображение $R_{t}: C_{1}(t) \rightarrow B_{2}$, причем для любого $v \in C_{1}(t) R_{t}(v)$ есть непрерывная функция аргумента $t \in\left[0, T_{0}\right]$.

Задачей Коши для оператора $R_{t}$ с начальным условием $\psi_{0} \in B_{1}$ называется система уравнений для функции $\psi(t), 0 \leqslant t \leqslant T_{0}$ :

$$
\frac{d}{d t} \psi(t)=R_{t}(\psi), \quad \psi(0)=\psi_{0},
$$

причем производная берется в норме $B_{2}$ :

$$
\lim _{\Delta t \rightarrow+0}\left\|\frac{d}{d t} \psi(t)-\frac{\psi(t+\Delta t)-\psi(t)}{\Delta t}\right\|_{2}=0 .
$$

Пусть задана образующая $T$-отображения $S_{t, \varepsilon}: C_{1}(t) \rightarrow C_{1}(t+\varepsilon)$.

ОПРЕДЕЛЕНИЕ 4 . Если при любом $t \in\left[0, T_{0}\right]$ образующая $S_{t, \varepsilon}$ удовлетворяет условию

$$
\lim _{\varepsilon \rightarrow+0}\left\|\frac{\left(S_{t, \varepsilon}[v]\right)(\varepsilon)-v(t)}{\varepsilon}-R_{t}(v)\right\|_{2}=0
$$

локально равномерно по $v \in C_{1}(t)$, то $S_{t, \varepsilon}$ называется инфинитезимальным разрешающим оператором для задачи Коши (22).

ЛЕмма 1. Пусть $S_{t, \varepsilon}-$ инфинитезималъный разрешающий оператор для задачи Коши (22), а Т-отображение $\psi(t)=\left(\prod \lim _{\tau=0}^{t} \circ S_{\tau, \partial \tau}^{\tau}\right)\left[\psi_{0}\right]$ существует на элементе $\psi_{0} \in B_{1}$ на отрезке времени $\left[0, T_{0}\right]$ в норме $C_{1}\left(T_{0}\right)$. Тогда функиия $\psi$ является решением задачи (22).

ДокАзАтЕЛьство. Пусть $\Delta_{N}-$ некоторое разбиение $\left[0, T_{0}\right]$ и

$$
\psi_{N}=S_{t_{N-1}, \Delta t_{N-1}} \circ \cdots \circ S_{t_{0}, \Delta t_{0}}\left[\psi_{0}\right]
$$

Тогда если $t_{j+1} \geqslant t \geqslant t_{j}$, то

$$
\psi_{N}(t)=\left(S_{t_{j}, t-t_{j}}\left[\psi_{N}\right]\right)(t)
$$

Поэтому в силу (23) найдется $N_{0}$ такое, что

$$
\lim _{\Delta t \rightarrow+0}\left\|\frac{\psi_{N}\left(t_{j}+\Delta t\right)-\psi_{N}\left(t_{j}\right)}{\Delta t}-R_{t_{j}}\left[\psi_{N}\right]\right\|_{2}=0
$$

равномерно по $N \geqslant N_{0}$.

Зафиксируем точку $t \in\left[0, T_{0}\right]$ и выберем последовательность разбиений $\left\{\Delta_{N}\right\}$ так, чтобы при каждом $N$ один из узлов $t_{j}$ совпадал с $t$. Тогда, в силу того, что оператор $R_{t}$ непрерывен и $T$-отображение с образующей $S_{t, \varepsilon}$ существует на $\psi_{0}$, имеем

$$
\lim _{\Delta t \rightarrow+0}\left\|\frac{\psi(t+\Delta t)-\psi(t)}{\Delta t}-R_{t}[\psi]\right\|_{2}=0 .
$$

Лемма доказана. 
Предположим теперь, что нелинейный оператор $R_{t}$ в задаче Коши $(23)$ имеет специальный вид

$$
R_{t}(\psi)=A_{t}(\psi) \psi(t)
$$

где $A_{t}(\psi)$ при каждом $\psi \in C_{1}(t)$ есть линейный непрерывный оператор из $B_{1}$ в $B_{2}$, являющийся инфинитезимальной образующей для однопараметрической полугруппы $e^{\varepsilon A_{t}(\psi)}$. В качестве инфинитезимального разрешающего оператора задачи

$$
\frac{d}{d t} \psi(t)=A_{t}(\psi) \psi(t), \quad \psi(0)=\psi_{0}
$$

можно выбрать оператор

$$
\left(S_{t, \varepsilon}[v]\right)(\tau)= \begin{cases}e^{(\tau-t) A_{t}(v)} v(t), & t \leqslant \tau \leqslant t+\varepsilon \\ v(t), & \tau \leqslant t\end{cases}
$$

$T$-отображение с такой образующей записывается следующим образом:

$$
\psi(t)=\prod_{\tau=0}^{t} e^{\tau A_{\tau}(\psi) d \tau} \psi_{0} .
$$

В случае, когда показатель экспоненты не зависит от $\psi$, это $T$-отображение переходит в $T$-произведение.

Проследим теперь аналогию между методом $T$-отображений и методом ломаных Эйлера. При решении обыкновенных дифференциальных уравнений в банаховом пространстве эта аналогия оказывается полной (см. ниже лемму 2).

Пусть $B$ - банахово пространство, $\Theta: B \times \mathbb{R} \rightarrow B$ - непрерывное отображение. Через $d x(t) / d t=\dot{x}^{k}(t)$ будем обозначать производную $\dot{r}(t)=\lim _{\Delta t \rightarrow 0}(x(t+\Delta t)-$ $x(t)) / \Delta t$ функции $t \rightarrow x(t) \in B$. Рассмотрим задачу Коши

$$
\dot{r}(t)=\Theta(x(t), t), \quad x(0)=x^{0} \in B .
$$

Построим $T$-отображение, отвечающее этой задаче. Образующую $S_{t, \varepsilon}$ зададим следующим образом:

$$
\left(S_{t, \varepsilon}[x]\right)(\tau)= \begin{cases}x(\tau), & \tau \leqslant t \\ x(t)+(\tau-t) \Theta(x(t), t), & t \leqslant \tau \leqslant t+\varepsilon .\end{cases}
$$

Образующая $S_{t, \varepsilon}$ является инфинитезимальным разрешающим оператором задачи Коши (27). Докажем существование $T$-отображения с этой образующей.

ОПРЕДЕЛЕНИЕ 5 . Отображение $\omega: B_{1} \rightarrow B_{2}$ банаховых пространств $B_{1}$ и $B_{2}$ будем называть липшиц-непрерывным, если существует такая непрерывная функция $\gamma$ на $\mathbb{R}^{2}$, что для любых $x, y \in B_{1}$

$$
\|\omega(x)-\omega(y)\|_{B_{2}} \leqslant\|x-y\|_{B_{1}} \gamma\left(\|x\|_{B_{1}},\|y\|_{B_{1}}\right) .
$$

Обозначим через $\|\cdot\|$ норму в банаховом пространстве $B$. 
ЛЕмма 2. Пусть отображение $\Theta$ липшиц-непрерывно и при любом $T>0$ существует константа $c \geqslant 0$ такая, что при $|t| \leqslant T$ и при любом $x \in B$ справедливо неравенство

$$
\|\Theta(x, t)\| \leqslant c(1+\|x\|) .
$$

Тогда T-отображение $x(t)=\prod \lim _{\tau=0}^{t} \circ \stackrel{\tau}{S}_{\tau, d \tau}\left[x^{0}\right]$ существует при любом $x^{0} \in B$ на любом отрезке $[0, T]$ и дает решение $x(t)$ задачи Коши (27). Это решение единственно.

Мы приведем подробное доказательство леммы 2. В монографии [19] показано, что это доказательство почти без изменений переносится на нелинейные интегродифференциальные уравнения, содержащие частные производные.

ДокАЗАТЕЛЬство. Пусть $0 \equiv t_{0}<t_{1}<\cdots<t_{N} \equiv t-$ некоторое разбиение отрезка $[0, t]$. Определим функцию $x^{(N)}$ на отрезке $[0, t]$ следующим равенством:

$$
x^{(N)}=S_{t_{N-1}, \Delta t_{N-1}} \circ \cdots \circ S_{t_{0}, \Delta t_{0}}\left[x^{0}\right] .
$$

На каждом отрезке $\tau \in\left[t_{k}, t_{k+1}\right], k=0,1, \ldots, N-1$, значения функции $x^{(N)}$ вычисляются по формуле

$$
x^{(N)}(\tau)=x^{(N)}\left(t_{k}\right)+\left(\tau-t_{k}\right) \Theta\left(x^{(N)}\left(t_{k}\right), t_{k}\right) .
$$

Таким образом, функция $x^{(N)}$ есть ломаная Эйлера для задачи $(27)$ в банаховом пространстве $B$. Сходимость $T$-отображения с образующей $S_{t, \varepsilon}$ означает существование предела $\lim _{\delta_{N} \rightarrow 0, N \rightarrow \infty} x^{(N)}$ последовательности ломаных Эйлера (когда диаметр разбиения $\delta_{N}=\sup \lim _{k} \Delta t_{k}$ стремится к нулю). Докажем существование этого предела.

Из формулы (30) и оценки (29) следует

$$
\left\|x^{(N)}(\tau)\right\| \leqslant\left\|x^{(N)}\left(t_{k}\right)\right\| .
$$

Константа $c$ не зависит от $\tau$ и выбора точек разбиения $t_{k}$. Из этого неравенства по индукции получим следующую оценку:

$$
\begin{aligned}
\left\|x^{(N)}(\tau)\right\| & \leqslant\left\|x^{0}\right\|\left(1+c \Delta t_{0}\right) \ldots\left(1+c \Delta t_{k-1}\right)\left(1+c\left(\tau-t_{k}\right)\right) \leqslant \\
& \leqslant\left\|x^{0}\right\| e^{\tau c} \leqslant\left\|x^{0}\right\| e^{t c} .
\end{aligned}
$$

Тем самым доказана равномерная ограниченность последовательности ломаных Эйлера. Из (28), (30) и (31) следует оценка разности

$$
\left\|x^{(N)}-x^{(N)}\left(t_{k}\right)\right\| \leqslant c\left(\tau-t_{k}\right)\left\|x^{(N)}\left(t_{k}\right)\right\| \leqslant c\left(\tau-t_{k}\right)\left\|x^{0}\right\| e^{t c} .
$$

Теперь докажем фундаментальность последовательности $\left\{x^{(N)}\right\}$ в пространстве $C([0, t], B)$. В силу (32) достаточно рассмотреть случай, когда точки $t_{k}$ в $(30)$ имеют вид $t_{k}=k t / N$.

Пусть $M=N l$, где $l$ - натуральное число. Достаточно доказать, что разность $x^{(N)}-x^{(N)}$ стремится к нулю в норме $C([0, t], B)$ при $N \rightarrow \infty$ равномерно по $l$. 
Обозначим $\varepsilon=t / M$. Пусть $\tau \in[0, t]$. Найдутся номер $k \in\{0,1, \ldots, N-1\}$ и номер $j \in\{0,1, \ldots, l-1\}$ такие, что

$$
t_{k}+j \varepsilon \leqslant \tau<t_{k}+(j+1) \varepsilon
$$

Из формулы (32) (записанной для функции $x^{(M)}$ вместо $\left.x^{(N)}\right)$ следует, что

$$
\begin{aligned}
x^{(M)}(\tau)= & x^{(M)}\left(t_{k}\right)+\sum_{s=0}^{j-1} \varepsilon \Theta\left(x^{(M)}\left(t_{k}+s \varepsilon\right), t_{k}+s \varepsilon\right)+ \\
& +\left(\tau-t_{k}-j \varepsilon\right) \Theta\left(x^{(M)}\left(t_{k}+j \varepsilon\right), t_{k}+j \varepsilon\right) .
\end{aligned}
$$

Вычтем из равенства (33) равенство (30). Оценим норму получившейся разности:

$$
\begin{aligned}
\left\|x^{(M)}(\tau)-x^{(N)}(\tau)\right\| \leqslant & \left\|x^{(M)}\left(t_{k}\right)-x^{(N)}\left(t_{k}\right)\right\|+ \\
& +\varepsilon \sum_{s=0}^{j}\left\|\Theta\left(x^{(M)}\left(t_{k}+s \varepsilon\right), t_{k}+s \varepsilon\right)-\Theta\left(x^{(N)}\left(t_{k}\right), t_{k}\right)\right\| .
\end{aligned}
$$

Как было показано в (31), последовательность $\left\{x^{(N)}\right\}$ равномерно ограничена на отрезке $[0, t]$. Отсюда и из липшиц-непрерывности отображения $\Theta$ следует оценка

$$
\begin{gathered}
\left\|\Theta\left(x^{(M)}\left(t_{k}+s \varepsilon\right), t_{k}+s \varepsilon\right)-\Theta\left(x^{(N)}\left(t_{k}\right), t_{k}\right)\right\| \leqslant \\
\leqslant c_{1}\left(\left\|x^{(M)}\left(t_{k}+s \varepsilon\right)-x^{(N)}\left(t_{k}\right)\right\|+s \varepsilon\right),
\end{gathered}
$$

где $c_{1}$ - некоторая константа, зависящая лишь от $t$ и от нормы $\left\|x^{0}\right\|$ начальной точки $x^{0}$.

Обозначим $\alpha(\tau)=\left\|x^{(M)}(\tau)-x^{(N)}(\tau)\right\|$. Используя формулы (35) и (32), перепишем неравенство (34) в следующем виде:

$$
\alpha(\tau) \leqslant \alpha\left(t_{k}\right)+\varepsilon \sum_{s=0}^{j} c_{1} \alpha\left(t_{k}+s \varepsilon\right)+c_{2} \varepsilon^{2} \frac{j(j+1)}{2},
$$

где

$$
c_{2}=c_{1} c\left\|x^{0}\right\| e^{t c}+c_{1}, \quad \varepsilon^{2} \frac{j(j+1)}{2} \leqslant \frac{\delta^{2}}{l^{2}} \cdot \frac{l(l+1)}{2} \leqslant \delta^{2} .
$$

Отсюда по индукции получается оценка

$$
\alpha(\tau) \leqslant\left[\alpha\left(t_{k}\right)\left(1+\varepsilon c_{1}\right)+c_{2} \delta^{2}\right]\left(1+\varepsilon c_{1}\right)^{l-1}, \quad \tau \in\left[t_{k}, t_{k+1}\right] .
$$

Снова применяя индукцию к этому неравенству, получим

$$
\begin{aligned}
\alpha(\tau) & \leqslant c_{2} \delta^{2}\left[\left(1+\varepsilon c_{1}\right)^{l}+\left(1+\varepsilon c_{1}\right)^{2 l}+\cdots+\left(1+\varepsilon c_{1}\right)^{N l}\right] \leqslant \\
& \leqslant N c_{2} \frac{t^{2}}{N^{2}}\left(1+\frac{t c_{1}}{M}\right)^{M} \leqslant \frac{c_{2} t^{2}}{N} e^{t c_{1}} .
\end{aligned}
$$

Таким образом, мы доказали, что $\alpha(\tau)=\left\|x^{(M)}(\tau)-x^{(N)}(\tau)\right\| \rightarrow 0$ при $N \rightarrow \infty$ равномерно по $l$ и по $\tau \in[0, t]$. Следовательно, последовательность $\left\{x^{(N)}\right\}$ фундаментальна в пространстве $C([0, t], B)$. Обозначим через $x(\tau)$ ее предел $x(\tau)=$ 
$\lim _{N \rightarrow \infty} x^{(N)}(\tau)$. Из (30) следует, что

$$
x^{(N)}\left(k \frac{t}{N}\right)=x^{0}+\sum_{s=0}^{k-1} \frac{t}{N} \Theta\left(x^{(N)}\left(s \frac{t}{N}\right), s \frac{t}{N}\right), \quad 1 \leqslant k \leqslant N .
$$

Пусть $0<\tau<t$. Положим в формуле $(36) k=[N \tau / t]$, где квадратные скобки обозначают целую часть. Учитывая, что $[N \tau / t](t / N) \rightarrow \tau$, получим из (36) интегральное уравнение для $x(\tau)$ :

$$
x(\tau)=x^{0}+\int_{0}^{\tau} \Theta(x(\mu), \mu) d \mu .
$$

Следовательно, функция $x(\tau)$ всюду на отрезке $[0, t]$ является решением задачи Коши (27). Единственность решения этой задачи следует из липшиц-непрерывности отображения $\tau$. Этим доказана сходимость $T$-отображения с образующей $S_{t, \varepsilon}$ к решению задачи $(27)$ при всех $t \in(0, \infty)$. Лемма доказана.

В заключение рассмотрим обобщение задачи (27). Пусть задано некоторое отображение $\Omega$ пространства $C(\mathbb{R}, B)$ в себя. Каждой непрерывной функции $x: \mathbb{R} \rightarrow B$ отображение $\Omega$ сопоставляет непрерывную функцию $\Omega[x]: \mathbb{R} \rightarrow B$. Предположим, что $\Omega$ удовлетворяет следующему условию:

(A) если $x, y \in C(\mathbb{R}, B)$ и $x(\tau)=y(\tau)$ при $0 \leqslant \tau \leqslant t$, то $\Omega[x](t)=\Omega[y](t)$.

Иными словами, значение $\Omega[x](t)$ не зависит от поведения функции $x=x(\tau)$ при $\tau>t$. Символически это можно записать так:

$$
\frac{\delta \Omega[x](t)}{\delta x(\tau)}=0 \quad \text { при } \quad \tau>t .
$$

Если $x \in C([0, T], B)$, то, доопределив функцию $x$ подходящей константой при $t>T$, мы получим непрерывную функцию на $\mathbb{R}$ (обозначив ее снова $x$ ) и, следовательно, сможем построить функцию $\Omega[x](\tau)$. Таким образом, отображение $\Omega$ индуцирует при каждом $T>0$ отображение $\Omega: C([0, T], B) \rightarrow C([0, T], B)$.

Рассмотрим задачу Коши

$$
\begin{aligned}
& \dot{x}(t)=\Omega[x](t), \quad t \in[0, T], \\
& x(0)=x^{0} \in B .
\end{aligned}
$$

Поскольку отображение $\Omega$ удовлетворяет условию (А), решение задачи (40) можно искать методом ломаных Эйлера. Каждому разбиению $0 \equiv t_{0}<t_{1}<\cdots<t_{N} \equiv T$ отрезка $[0, T]$ сопоставим непрерывную функцию $x^{(N)}$ на $[0, T]$ (ломаную Эйлера), которая определяется рекуррентной формулой

$$
\begin{aligned}
& x^{(N)}(0)=x^{0}, \\
& x^{(N)}(t)=x^{(N)}\left(t_{k}\right)+\left(t-t_{k}\right) \Omega\left[x^{(N)}\right]\left(t_{k}\right), \quad t \in\left[t_{k}, t_{k+1}\right] .
\end{aligned}
$$

Предел $\lim _{N \rightarrow \infty} x^{(N)}(t)=x(t)$, если он существует, можно представить в виде $T$-отображения:

$$
x(t)=\left(\prod_{\tau=0}^{t} \circ{ }^{\tau} S_{\tau, d \tau}[x]\right)\left(x^{0}\right)
$$


где образующая $S_{t, \varepsilon}$ задана формулой

$$
\left(S_{t, \varepsilon}[x]\right)(\tau)= \begin{cases}x(\tau), & \tau \leqslant t \\ x(\tau)+(\tau-t) \Omega[x](t), & t \leqslant \tau \leqslant t+\varepsilon .\end{cases}
$$

Лемма 3. А. Пусть отображение $\Omega$ пространства $C([0, R], B)$ в себя при каждом $R>0$ липшич-непрерывно. Тогда найдется отрезок $\left[0, T_{0}\right]$, на котором решение задачи Коши (37) существует, единственно и равно Т-отображению (38).

Б. Если справедливо условие утверждения $A$ и отображение $\Omega$ при каждом $R>0$ удовлетворяет оценке

$$
\|\Omega[x]\|_{C([0, R], B)} \leqslant \operatorname{const}_{R}\left(1+\|x\|_{C([0, R], B)}\right),
$$

то Т-отображение (38) существует на любом отрезке $[0, T]$ и равно решению задачи (37).

Доказательство леммы почти дословно повторяет доказательство леммы 2 и мы его опускаем.

3.2. Уравнение Власова и его обобщение. Здесь и ниже мы будем рассматривать лишь вещественные функции, не оговаривая этого обстоятельства каждый раз. Введем обозначение для скалярного произведения:

$$
\frac{\partial a}{\partial p} \frac{\partial b}{\partial q}=\sum_{k=1}^{n} \frac{\partial a}{\partial p_{k}} \frac{\partial b}{\partial q_{k}}
$$

Пусть

$$
\mathcal{H}(q, p,[F])=H_{0}(q, p)+\iint V\left(q, p, q^{\prime}, p^{\prime}\right) F\left(q^{\prime}, p^{\prime}, t\right) d q^{\prime} d p^{\prime},
$$

где $q, p \in \mathbb{R}^{n}, t \in \mathbb{R}, H_{0}$ и $V$ - некоторые гладкие функции.

Рассмотрим следующее нелинейное уравнение (уравнение Власова):

$$
\frac{\partial F}{\partial t}+\frac{\partial \mathcal{H}}{\partial p}(q, p,[F]) \frac{\partial F}{\partial q}-\frac{\partial \mathcal{H}}{\partial q}(q, p,[F]) \frac{\partial F}{\partial p}=0 .
$$

Ниже мы докажем (см. теорему 3), что задача Коши для уравнения Власова обладает следующим свойством: ее решения $F\left(q, p, t^{\prime}\right)$ и $F\left(q, p, t^{\prime \prime}\right)$ в любые два момента времени $t^{\prime}$ и $t^{\prime \prime}$ связаны между собой каноническим преобразованием. Это фундаментальное свойство сохраняется и для существенно более общих уравнений. Напомним, что диффеоморфизм $\gamma: \mathbb{R}^{2 n} \rightarrow \mathbb{R}^{2 n}$ называется каноническим преобразованием, если он сохраняет форму $\Omega$, т.е. $\gamma^{*} \Omega=\Omega$. Через $\gamma^{*}$ здесь обозначено отображение дифференциальных форм, индуцированное $\gamma$.

Введем ряд обозначений, которые мы будем использовать в дальнейшем. Обозначим через $C^{k}\left(\mathbb{R}^{m} \rightarrow \mathbb{R}^{n}\right)$ пространство $k$ раз дифференцируемых отображений $v: \mathbb{R}^{m} \rightarrow \mathbb{R}^{n}$, наделенное следующей нормой:

$$
\|v\|_{C^{(k)}\left(\mathbb{R}^{m} \rightarrow \mathbb{R}^{n}\right)}=\sup \lim _{x \in \mathbb{R}^{m}}\left\{\sum_{|\alpha|=1}^{k}\left|\left(\frac{\partial}{\partial x}\right)^{\alpha} v(x)\right|\right\}+\sup \lim _{x \in \mathbb{R}^{m}} \frac{|v(x)|}{1+|x|} .
$$


Пусть $C^{\infty}\left(\mathbb{R}^{m} \rightarrow \mathbb{R}^{n}\right) \bigcap \lim _{k} C^{(k)}\left(\mathbb{R}^{m} \rightarrow \mathbb{R}^{n}\right)$ - соответствующее счетно-нормированное пространство гладких отображений. Кроме того, введем шкалу гильбертовых пространств $H_{s}^{l}\left(\mathbb{R}^{m}\right)$, каждое из которых является пополнением пространства Шварца $S\left(\mathbb{R}^{m}\right)$ по норме

$$
\|u\|_{H_{s}^{l}}=\left(\int\left(1+|x|^{2}\right)^{-l}\left|(1-\Delta)^{s / 2} u(x)\right|^{2} d x\right)^{1 / 2} .
$$

Пространство

$$
S_{-\infty}\left(\mathbb{R}^{m}\right) \stackrel{\text { def }}{=} \bigcup \lim _{s} \bigcap \lim _{l} H_{s}^{l}\left(\mathbb{R}^{m}\right)
$$

наделим естественной сходимостью: скажем, что $u_{n} \rightarrow 0$, если $\exists s \forall l\left\|u_{n}\right\|_{H_{s}^{l}} \rightarrow 0$.

Двойственное к $S_{-\infty}$ пространство обозначим

$$
S^{\infty}\left(\mathbb{R}^{m}\right) \stackrel{\text { def }}{=} \bigcup \lim _{l} \bigcap \lim _{s} H_{s}^{l}\left(\mathbb{R}^{m}\right)
$$

и наделим его следующей сходимостью: $u_{n} \rightarrow 0$, если $\exists l \forall k=0,1, \ldots$

$$
\left\|u_{n}\right\|_{S_{l}^{k}} \stackrel{\text { def }}{=} \sup \lim _{x}\left\{(1+|x|)^{-l} \sum_{|\alpha|=0}^{k}\left|\left(\frac{\partial}{\partial x}\right)^{\alpha} u_{n}(x)\right|\right\} \rightarrow 0 .
$$

И, наконец, последний объект, который нам понадобится, - фазовое пространство $\mathbb{R}^{2 n}$, т.е. четномерное евклидово пространство, наделенное симплектической структурой, которую задает невырожденная 2-форма $\Omega=\frac{1}{2} J d z \wedge d z$, где $z \in \mathbb{R}^{2 n}$,

$$
J=\left(\begin{array}{cc}
0 & I \\
-I & 0
\end{array}\right)
$$

$I$ - единичная $(n \times n)$-матрица. Скобка Пуассона двух функций $H, f$ на $\mathbb{R}^{2 n}$ задается формулой $\{H, f\}=\left\langle J \frac{\partial H}{\partial Z}, \frac{\partial f}{\partial z}\right\rangle$, где $\langle\cdot, \cdot\rangle$ - скалярное произведение в $\mathbb{R}^{2 n}$.

Рассмотрим теперь некоторое отображение

$$
\begin{aligned}
& H: S_{-\infty}\left(\mathbb{R}^{2 n}\right) \times C^{\infty}\left(\mathbb{R}^{2 n} \rightarrow \mathbb{R}^{2 n}\right) \rightarrow S^{\infty}\left(\mathbb{R}^{2 n}\right), \\
& H:(f, v) \rightarrow H[f, v] .
\end{aligned}
$$

При фиксированных $f$ и $v$ функция $H[f, v]$ есть гладкая функция на фазовом пространстве $\mathbb{R}^{2 n}$, ее значение в точке $z$ обозначим $H[f, v](z)$. Везде ниже будем предполагать, что мнимая часть $H[f, v]$ равна нулю, если мнимая часть $f$ равна нулю.

ОПРЕДЕЛЕНИЕ 6. Отображение $H$ назовем отображением Гамильтона ${ }^{4)}$, если оно инвариантно относительно канонических преобразований, т.е. $H\left[\gamma^{*} f, v \circ \gamma\right]=H[f, v]$ для любого канонического преобразования $\gamma \in C^{\infty}\left(\mathbb{R}^{2 n} \rightarrow \mathbb{R}^{2 n}\right)$.

ПримеР 5. Положим

$$
H[f, v](z)=H_{0}(z)+\int V\left(z, v\left(z^{\prime}\right)\right) f\left(z^{\prime}\right) d z^{\prime}
$$

\footnotetext{
4) По аналогии с термином "функция Гамильтона” в классической механике. Ниже мы увидим, что отображение Гамильтона описывает движение частиц в самосогласованном поле.
} 
где $H_{0} \in S^{\infty}\left(\mathbb{R}^{2 n}\right), V \in S\left(\mathbb{R}^{4 n}\right)$ - некоторые вещественные функции. Отображение $H$, определенное этой формулой, является отображением Гамильтона.

Пусть id - тождественное отображение $\mathbb{R}^{2 n}$ на себя. Отображению Гамильтона $H$ сопоставим уравнение

$$
\frac{\partial F}{\partial t}+\left\langle J \frac{\partial H[F, \mathrm{id}]}{\partial z}, \frac{\partial F}{\partial z}\right\rangle=0
$$

и назовем его уравнением Власова-Лиувилля. В случае, когда отображение Гамильтона $H$ задано формулой (41), уравнение Власова-Лиувилля совпадает с уравнением Власова (39), поскольку в этом случае $H[F, \mathrm{id}](z)=\mathcal{H}(q, p,[F])$, где $z=(q, p)$, $q \in \mathbb{R}^{n}, p \in \mathbb{R}^{n}$.

Общее уравнение Власова-Лиувилля (42) будет занимать центральное место в последующих рассуждениях. Здесь мы получим теорему существования решения задачи Коши для этого уравнения и проследим его аналогию с классическим уравнением Лиувилля.

Сопоставим отображению Гамильтона $H$ и некоторой вещественной функции $F_{0} \in$ $S_{-\infty}\left(\mathbb{R}^{2 n}\right)$ следующую задачу Коши:

$$
\dot{Z}=J \frac{\partial H\left[F_{0}, Z\right]}{\partial z} \circ Z,\left.\quad Z\right|_{t=0}=\mathrm{id}
$$

в пространстве $C^{\infty}\left(\mathbb{R}^{2 n} \rightarrow \mathbb{R}^{2 n}\right)$. Уравнение $(43)$ назовем уравнением Власова-Гамильтона.

Решением задачи (43) является семейство гладких отображений $Z[t]: \mathbb{R}^{2 n} \rightarrow \mathbb{R}^{2 n}$, совпадающее при $t=0$ с тождественным отображением. Значение отображения $Z[t]$ в точке $w \in \mathbb{R}^{2 n}$ будем обозначать $Z(w, t)=Z[t](w)$.

Правая часть дифференциального уравнения задачи (43) есть отображение $J \frac{\partial H\left[F_{0}, Z[t]\right]}{\partial z} \circ Z[t]$, принимающее в точке $w$ значение $J \frac{\partial H\left[F_{0}, Z[t]\right]}{\partial z}(Z(w, t))$. В этих обозначениях задача (43) имеет вид

$$
\frac{\partial Z(w, t)}{\partial t}=J \frac{\partial H\left[F_{0}, Z[t]\right]}{\partial z}(Z(w, t)), \quad Z(w, 0)=w .
$$

Пример 6. Выпишем уравнение Власова-Гамильтона в том случае, когда отображение $H$ задано формулой (41). Из (43) имеем

$$
\frac{\partial Z(w, t)}{\partial t}=J \frac{\partial H_{0}}{\partial z}(Z(w, t))+\int J \frac{\partial V}{\partial Z}\left(Z(w, t) Z\left(w^{\prime}, t\right)\right) f\left(w^{\prime}\right) d w^{\prime}
$$

Это интегродифференциальное уравнение в $\mathbb{R}^{2 n}$. Начальное условие имеет вид $Z(w, 0)=w$. Мы рассматриваем уравнение (44) как обыкновенное дифференциальное уравнение в пространстве отображений $C^{\infty}\left(\mathbb{R}^{2 n} \rightarrow \mathbb{R}^{2 n}\right)$.

Предположим теперь, что отображение Гамильтона $H$ и функция $F_{0}$ удовлетворяют следующим двум условиям.

УсловиЕ 1. Отображение $v \rightarrow \partial H\left[F_{0}, v\right] / \partial z$ расширяется до липшиц-непрерывного отображения пространства $C\left(\mathbb{R}^{2 n} \rightarrow \mathbb{R}^{2 n}\right)$ в себя. 
УСловие 2. Существуют константы $c_{\alpha} \geqslant 0$ такие, что для всех $z \in \mathbb{R}^{2 n}, v \in$ $C\left(\mathbb{R}^{2 n} \rightarrow \mathbb{R}^{2 n}\right)$ выполнены неравенства

$$
\left|\left(\frac{\partial}{\partial z}\right)^{\alpha} H\left[F_{0}, v\right](z)\right| \leqslant c_{\alpha} \quad \text { при }|\alpha| \geqslant 2 .
$$

Если отображение $H$ задано формулой $(41)$, а функция $F_{0}$ порождает меру $F_{0}(z) d z$ с компактным носителем в $\mathbb{R}^{2 n}$, то условия 1,2 будут выполнены при следующих ограничениях на функции $H_{0}$ и $V$ :

$$
\begin{gathered}
\left|\frac{\partial H_{0}}{\partial z}(z)\right|+\left|\left(\frac{\partial}{\partial z}\right) V\left(z, z^{\prime}\right)\right| \leqslant c_{1}(1+|z|) \\
\left|\left(\frac{\partial}{\partial z}\right)^{\alpha} H_{0}(z)\right|+\left|\left(\frac{\partial}{\partial z}\right)^{\alpha} V\left(z, z^{\prime}\right)\right| \leqslant c_{\alpha}, \quad|\alpha| \geqslant 2 .
\end{gathered}
$$

Как мы увидим ниже, при построении квазиклассических асимптотик, функция $F_{0}$ имеет вид $F_{0}(z)=|\varphi(z)|^{2} \delta_{\Lambda}(z)$, где $\varphi \in C_{0}^{\infty}$, а $\delta_{\Lambda}$ есть $\delta$-функция, сосредоточенная на лагранжевом подмногообразии $\Lambda \in \mathbb{R}^{2 n}$.

Покажем, что условия 1, 2 обеспечивают существование решения задачи (43) при Bcex $t \in \mathbb{R}$.

Теорема 2. Если отображсение Гамильтона $H$ и функиия $F_{0}$ удовлетворяют условиям 1, 2, то решение $Z[t]$ задачи (43) существует в $C^{\infty}\left(\mathbb{R}^{2 n} \rightarrow \mathbb{R}^{2 n}\right)$ при всех $t$. Это решение единственно и является каноническим преобразованием фазового пространства $\mathbb{R}^{2 n}$.

ДокАЗАтЕльство. Определим отображение $\Xi$ пространства $C\left(\mathbb{R}^{2 n} \rightarrow \mathbb{R}^{2 n}\right)$ в себя следующей формулой:

$$
\Xi(v) \stackrel{\text { def }}{=} \frac{\partial H\left[F_{0}, v\right]}{\partial z} \circ v
$$

Задача (43) имеет вид

$$
\dot{Z}=\Xi(Z), \quad Z[0]=\mathrm{id} .
$$

Докажем разрешимость (45) в пространстве $C\left(\mathbb{R}^{2 n} \rightarrow \mathbb{R}^{2 n}\right)$. Имеем оценку в норме $C\left(\mathbb{R}^{2 n} \rightarrow \mathbb{R}^{2 n}\right):$

$$
\|\Xi(v)\| \leqslant \operatorname{const}(1+\|v\|) .
$$

Кроме того, в силу условий 1,2 отображение $\Xi$ пространства $C\left(\mathbb{R}^{2 n} \rightarrow \mathbb{R}^{2 n}\right)$ в себя липшиц-непрерывно. Лемма 2 гарантирует в этом случае однозначную разрешимость задачи (45) в пространстве $C\left(\mathbb{R}^{2 n} \rightarrow \mathbb{R}^{2 n}\right)$ при всех $t \in \mathbb{R}$. Пусть $Z[t](w)=Z(w, t)$ - значение решения задачи (45) в точке $w$. Мы имеем

$$
\frac{\partial Z}{\partial t}(w, t)=f(Z(w, t), t), \quad Z(w, 0)=w \in \mathbb{R}^{2 n},
$$

где

$$
f(z, t) \stackrel{\text { def }}{=} \Xi[Z[t]](z), \quad f(z, t) \leqslant a_{0}(t)<\infty .
$$

Из (46) дифференцированием по $w$ получим

$$
\frac{\partial}{\partial t}\left(\frac{\partial Z}{\partial w}\right)=\frac{\partial f}{\partial Z}(Z, t) \frac{\partial Z}{\partial w},\left.\quad \frac{\partial Z}{\partial w}\right|_{t=0}=\mathrm{id} .
$$


Следовательно,

$$
\frac{\partial Z(w, t)}{\partial w}=\prod_{\mu=0}^{t} \exp \left\{\frac{\mu}{\frac{\partial f}{\partial z}(Z(w, \mu), \mu) d \mu}\right\} .
$$

Отсюда получаем оценку:

$$
\left|\frac{\partial Z}{\partial w}(w, t)\right| \leqslant \exp \left\{\int_{0}^{t}\left|\frac{\partial f}{\partial z}(Z(w, \mu), \mu)\right| d \mu\right\} \leqslant \exp \left\{\int_{0}^{t} a_{0}(\mu) d \mu\right\} .
$$

Таким образом, доказано, что $Z[t] \in C^{(1)}\left(\mathbb{R}^{2 n} \rightarrow \mathbb{R}^{2 n}\right)$. Точно так же, используя индукцию, можно доказать, что $Z[t] \in C^{(k)}\left(\mathbb{R}^{2 n} \rightarrow \mathbb{R}^{2 n}\right)$ для любого $k=$ $0,1,2, \ldots$. Следовательно, $Z[t]$ - это единственное решение задачи (45) в пространстве $C^{\infty}\left(\mathbb{R}^{2 n} \rightarrow \mathbb{R}^{2 n}\right)$.

Обозначим $L(z, t)=H\left[F_{0}, Z[t]\right](z)$ и перепишем задачу (43) (или (45)) следующим образом:

$$
\dot{Z}=J \frac{\partial L}{\partial Z}(Z, t),\left.\quad Z\right|_{t=0}=\mathrm{id} .
$$

Решением этой задачи Коши, как хорошо известно, является семейство канонических преобразований. Поэтому $Z[t]$ канонично. Теорема доказана.

СлЕДСТВИЕ 2. Пусть $\Lambda_{0}$ - лагранжево многообразие в фазовом пространстве $\mathbb{R}^{2 n}$ (но на нем форма $p d q$ замкнута). Тогда многообразие $\Lambda_{t} \stackrel{\text { def }}{=} Z[t] \Lambda_{0}=\{Z(w, t) \mid$ $\left.w \in \Lambda_{0}\right\}$, полученное сдвигом $\Lambda_{0}$ вдоль решений уравнения Власова-Гамильтона, также лагранжево.

ЗАмЕчАниЕ 5. Построенное в теореме 2 семейство канонических преобразований $Z[t]$ не является, вообще говоря, группой преобразований, т.е. $Z\left[t_{1}+t_{2}\right] \neq Z\left[t_{1}\right] Z\left[t_{2}\right]$. Однако если функция $F_{0}$ такова, что $H\left[Z[t]^{*} F_{0}, v\right]=H\left[F_{0}, v\right]$ для всех $t \in \mathbb{R}$, $v \in C^{\infty}\left(\mathbb{R}^{2 n} \rightarrow \mathbb{R}^{2 n}\right)$, то $Z[t]$ - группа. В частности, это так, если функция $F_{0}$ есть $\delta$-функция, сосредоточенная на лагранжевом многообразии, инвариантном относительно всех преобразований $Z[t]$.

Рассмотрим теперь уравнение Власова-Лиувилля (42) и поставим для него задачу Коши:

$$
\frac{\partial F}{\partial t}+\left\langle J \frac{\partial H[F, \mathrm{id}]}{\partial z}(z), \frac{\partial F}{\partial z}\right\rangle=0,\left.\quad F\right|_{t=0}=F_{0} .
$$

Теорема 3. Пусть отображсение Гамильтона $H$ и функиия $F_{0}$ удовлетворяют условиям 1, 2. Тогда решение $F(t) \in S_{-\infty}\left(\mathbb{R}^{2 n}\right)$ задачи (47) существует и имеет вид

$$
F(t)=\left(Z[t]^{-1}\right)^{*} F_{0},
$$

где $Z[t]$ - семейство канонических преобразований, заданное уравнениями (43).

ДокАзАтельство. Определим функцию $F(t)$ равенством (48). Так как отображение $H$ инвариантно относительно канонических преобразований, то

$$
H\left[F_{0}, Z[t]\right]=H\left[Z[t]^{-1 *} F_{0}, \mathrm{id}\right]=H[F(t), \mathrm{id}] .
$$

Далее, поскольку

$$
F_{0}(w)=Z[t]^{*} F(t)
$$


то

$$
\frac{\partial}{\partial t}\left[Z[t]^{*} F(t)\right]=0
$$

или

$$
Z[t]^{*}\left(\frac{\partial F}{\partial t}+\left\langle\dot{Z}, \frac{\partial F}{\partial z}\right\rangle\right)=0 .
$$

Используя (43) и (49), заменим здесь $\dot{Z}$ на $J \frac{\partial H[F, \mathrm{id}]}{\partial z}$. Получим

$$
Z[t]^{*}\left(\frac{\partial F}{\partial t}\left\langle J \frac{\partial H[F, \mathrm{id}]}{\partial z}, \frac{\partial F}{\partial z}\right\rangle\right)=0 .
$$

Следовательно, функция $F$ удовлетворяет дифференциальному уравнению (47). Начальное условие (47) также выполнено, так как $Z[0]=\mathrm{id}$. Теорема доказана.

С помощью теоремы 3 решение задачи (47) иногда удается построить явно. Рассмотрим пример.

ПримеР 7. Решим следующую задачу Коши для уравнения Власова:

$$
\begin{gathered}
\frac{\partial F}{\partial t}+p \frac{\partial F}{\partial q}-q \frac{\partial F}{\partial q}-\frac{\partial F}{\partial q} \iint q^{\prime} F\left(q^{\prime}, p^{\prime}, t\right) d q^{\prime} d p^{\prime}=0 \\
\left.F(q, p, t)\right|_{t=0}=\frac{1}{\sqrt{\pi}} e^{-q^{2}} \delta(p-\cos q)
\end{gathered}
$$

где $\delta-\delta$-функция Дирака. Напишем уравнения Власова-Гамильтона, отвечающие задаче $(53)$ при $z=(q, p), Z[t]=(Q[t], P[t]), Z(z, t)=(Q(z, t), P(z, t))$ :

$$
\begin{gathered}
\dot{Q}=P, \quad \dot{P}=-Q-\frac{1}{\sqrt{\pi}} \int Q\left(q_{0}, \cos q_{0}, t\right) e^{-q_{0}^{2}} d q_{0}, \\
\left.Q(q, p, t)\right|_{t=0}=q,\left.\quad P(q, p, t)\right|_{t=0}=p .
\end{gathered}
$$

Для функции $Q$ получаем уравнение второго порядка:

$$
\ddot{Q}+Q+f(t)=0, \quad Q(0)=q, \quad \dot{Q}(0)=p,
$$

где

$$
f(t)=\frac{1}{\sqrt{\pi}} \int Q(q, \cos q, t) e^{-q^{2}} d q .
$$

Решение этого уравнения имеет вид

$$
Q(q, p, t)=q \cos t+p \sin t-\int_{0}^{t} \sin (t-\tau) f(\tau) d \tau
$$

Положим $p=\cos q$, затем умножим равенство (55) на $\frac{1}{\sqrt{\pi}} e^{-q^{2}}$ и проинтегрируем по $d q$. Учитывая, что

$$
\int q e^{-q^{2}} d q=0, \quad \int e^{-q^{2}} d q=\sqrt{\pi}, \quad \int \cos q \cdot e^{-q^{2}} d q=\frac{\sqrt{\pi}}{\sqrt[4]{e}}
$$

получим

$$
f(t)=\frac{1}{\sqrt[4]{e}} \sin t-\int_{0}^{t} \sin (t-\tau) f(\tau) d \tau
$$

6 Теоретическая и математическая физика, т. 161, № 3, 2009 г. 
Отсюда

$$
\ddot{f}+2 f=0, \quad f(0)=0, \quad \ddot{f}(0)=\frac{1}{\sqrt[4]{e}} .
$$

Решая это уравнение, найдем $f(t)$, а из (55) найдем функцию $Q$ :

$$
Q(q, p, t)=q \cos t+p \sin t+\frac{1}{\sqrt[4]{e}}\left(\frac{\sin t \sqrt{2}}{\sqrt{2}}-\sin t\right) .
$$

Функция $P$ определяется из уравнения $\dot{Q}=P$. Из общей формулы (48) теперь получим решение задачи (53):

$$
F(q, p, t)=\frac{1}{\sqrt{\pi}} e^{-q_{0}^{2}(q, p, t)} \delta\left(p_{0}(q, p, t)-\cos q_{0}(q, p, t)\right),
$$

где

$$
\begin{aligned}
& q_{0}(q, p, t)=q \cos t-p \sin t+\frac{\sin t}{\sqrt[4]{e}}(\cos t \sqrt{2}-\cos t)-\frac{\cos t}{\sqrt[4]{e}}\left(\frac{\sin t \sqrt{2}}{\sqrt{2}}-\sin t\right) \\
& p_{0}(q, p, t)=q \sin t+p \cos t-\frac{\cos t}{\sqrt[4]{e}}(\cos t \sqrt{2}-\cos t)-\frac{\sin t}{\sqrt[4]{e}}\left(\frac{\sin t \sqrt{2}}{\sqrt{2}}-\sin t\right) .
\end{aligned}
$$

Введем теперь два новых понятия - действие и энтропию для уравнения Власова-Лиувилля.

Пусть $\Lambda_{0}=\{q(\alpha), p(\alpha)\}$ - некоторое лагранжево многообразие в $\mathbb{R}^{2 n}=\mathbb{R}_{q}^{n} \times \mathbb{R}_{p}^{n}$, $\alpha$ - локальные координаты на $\Lambda_{0}$. Предположим, что $\Lambda_{0}$ односвязно. Пусть $d \mu_{0}-$ некоторая мера на $\Lambda_{0}$, а $F_{0}$ - обобщенная функция на $\mathbb{R}^{2 n}$, сосредоточенная на $\Lambda_{0}$ и определяемая мерой $\mu_{0}$ :

$$
\left\langle F_{0}, \varphi\right\rangle \stackrel{\text { def }}{=} \int_{\Lambda_{0}} \varphi(q(\alpha), p(\alpha)) d \mu_{0}(\alpha), \quad \varphi \in C\left(\mathbb{R}^{2 n}\right) .
$$

Предположим, что функция $F_{0}$ и отображение Гамильтона $H$ удовлетворяют условиям 1,2 . Построим решение $F(t)=\left(Z[t]^{-1}\right)^{*} F_{0}$ задачи Коши (47) для уравнения Власова-Лиувилля, где $Z[t]=(Q[t], P[t])$ - каноническое преобразование, заданное уравнениями Власова-Гамильтона (43). Это преобразование переводит $\Lambda_{0}$ в некоторое лагранжево многообразие $\Lambda_{t}=Z[t]\left(\Lambda_{0}\right)$. Мера $d \mu_{0}$ индуцирует меру $d \mu_{t}=\left(Z[t]^{-1}\right)^{*} d \mu_{0}$ на $\Lambda_{t}$, так что для любой непрерывной функции $\chi$ на $\Lambda_{0}$ справедливо равенство

$$
\int_{\Lambda_{0}} \chi d \mu_{0}=\int_{\Lambda_{t}}\left(Z[t]^{-1}\right)^{*} \chi d \mu_{t}
$$

Поскольку многообразие $\Lambda_{0}$, по предположению, односвязно, на нем существует глобальное действие $S_{0}$, т.е. такая гладкая функция, для которой

$$
d S_{0}=\left.p d q\right|_{\Lambda_{0}}
$$

Этим условием действие $S_{0}$ определено с точностью до произвольной аддитивной константы. Рассмотрим далее при каждом $t$ следующую функцию $\widetilde{S}(t)$ на $\Lambda_{0}$ :

$$
\widetilde{S}(t)=S_{0}+\int_{0}^{t}\left(P[\tau] \dot{Q}[\tau]-H\left[F_{0}, Q[\tau], P[\tau]\right](Q[\tau], P[\tau])\right)_{\Lambda_{0}} d \tau
$$


ОПРЕДЕЛЕНИЕ 7. Функцию

$$
S(t) \stackrel{\text { def }}{=}\left(Z[t]^{-1}\right)^{*} \widetilde{S}(t)
$$

будем называть действием, отвечающим задаче Коши (47) для уравнения ВласоваЛиувилля. Энтропией $H_{0}(t)$ этой задачи будем называть среднее от действия по мере $d \mu_{t}$ :

$$
H_{0}(t) \stackrel{\text { def }}{=} \int_{\Lambda_{t}} S(t) d \mu_{t}
$$

ПримеР 8. Рассмотрим многообразие $\Lambda_{0}$, заданное уравнением

$$
p=\frac{\partial \Phi_{0}}{\partial q}(q)
$$

где $\Phi_{0} \in C_{0}^{\infty}\left(\mathbb{R}^{n}\right)$. Заметим, что в этом случае переменные $q=\left(q_{1}, \ldots, q_{n}\right)$ могут служить глобальными координатами на $\Lambda_{0} \subset \mathbb{R}_{q}^{n} \times \mathbb{R}_{q}^{n}$. Рассмотрим начальное распределение, сосредоточенное на $\Lambda_{0}$ с весом $\kappa_{0} \in C_{0}^{\infty}\left(\mathbb{R}^{n}\right)$ :

$$
F_{0}(q, p)=\kappa_{0}(q) \delta\left(p-\frac{\partial \Phi_{0}}{\partial q}(q)\right)
$$

т.е. $d \mu_{0}=\kappa_{0} d q$. Мы вновь обозначим через $F(q, p, t)$ решение задачи Коши $(47)$ с начальным условием (60). Для энтропии этой задачи Коши на том отрезке времени $t \in[0, T]$, на котором проекция $\pi_{q}: \Lambda_{t} \rightarrow \mathbb{R}_{q}^{n}$ многообразия $\Lambda_{t}$ на координатную $q$-гиперплоскость является диффеоморфизмом (т.е. вплоть до фокальной точки), имеет место следующая формула:

$$
H_{0}(t)=\iint \Phi(q, t) F(q, p, t) d q d p
$$

где $\Phi$ - решение задачи Коши

$$
\frac{\partial \Phi}{\partial t}+H[F(t), \mathrm{id}]\left(q, \frac{\partial \Phi}{\partial q}\right)=0,\left.\quad \Phi\right|_{t=0}=\Phi_{0}
$$

Докажем это утверждение. Обозначим через $(Q(q, p, t), P(q, p, t))$ значение отображения $Z[t]=(Q[t], P[t])$ в точке $(q, p) \in \mathbb{R}^{2 n}$. Функции

$$
X\left(q^{0}, t\right) \stackrel{\text { def }}{=} Q\left(q^{0}, \frac{\partial \Phi_{0}}{\partial q}\left(q^{0}\right), t\right), \quad \Xi(q, t)=P\left(q^{0}, \frac{\partial \Phi_{0}}{\partial q}\left(q^{0}\right), t\right)
$$

удовлетворяют следующей гамильтоновой системе уравнений:

$$
\begin{array}{ll}
\dot{X}=\frac{\partial H[F(t), \mathrm{id}]}{\partial p}(X, \Xi), & \left.X\right|_{t=0}=q_{0}, \\
\dot{\Xi}=-\frac{\partial H[F(t), \mathrm{id}]}{\partial q}(X, \Xi), & \left.\Xi\right|_{t=0}=\frac{\partial \Phi_{0}}{\partial q}\left(q^{0}\right) .
\end{array}
$$


С другой стороны, по определению (57), (58), имеем

$$
\begin{aligned}
\left(\pi_{q}^{-1}\right)^{*} S(t)= & {\left[\Phi_{0}\left(q^{0}\right)+\int_{0}^{t}\left(\Xi\left(q^{0}, \tau\right) \dot{X}\left(q_{0}, \tau\right)-\right.\right.} \\
& \left.\left.-H[F(\tau), \operatorname{id}]\left(X\left(q^{0}, \tau\right), \Xi\left(q^{0}, \tau\right)\right)\right) d \tau\right]_{q^{0}=q^{0}(q, t)}
\end{aligned}
$$

где $q^{0}(q, t)$ - решение неявного уравнения $q=X\left(q^{0}, t\right)$. Через $\pi_{q}^{-1}$ мы обозначили отображение, обратное к проекции $\pi_{q}: \Lambda_{t} \rightarrow \mathbb{R}_{q}^{n}$. В силу предположения о невырожденности этой проекции такое обратное отображение существует.

Хорошо известно, что функция $\Phi(q, t)=\left(\pi_{q}^{-1}\right)^{*} S(t)$, заданная формулой (63), является решением задачи (62). Остается доказать, что энтропия вычисляется по формуле (61).

Преобразуем интеграл в правой части (61):

$$
\begin{aligned}
\iint \Phi(q, t) F(q, p, t) d q d p & =\iint\left[\left(\pi_{q}^{-1}\right)^{*}\left(Z[t]^{-1}\right)^{*} \widetilde{S}(t)\right]\left[\left(Z[t]^{-1}\right)^{*} F_{0}\right] d p d q= \\
& =\left.\int \chi_{0}\left(q^{0}\right)\left[Z[t]^{*}\left(\pi_{q}^{-1}\right)^{*}\left(Z[t]^{-1}\right)^{*} \widetilde{S}(t)\right]\right|_{p^{0}=\frac{\partial \Phi}{\partial q}\left(q^{0}\right)} d q .
\end{aligned}
$$

Функция, стоящая в квадратных скобках в последнем интеграле, равна

$$
\left(\pi_{q^{0}}^{-1}\right)^{*}\left[\pi_{q^{0}}^{*} Z[t]^{*}\left(\pi_{q}^{-1}\right)^{*}\left(Z[t]^{-1}\right)^{*} \widetilde{S}(t)\right]=\left(\pi_{q^{0}}^{-1}\right)^{*} \widetilde{S}(t),
$$

где $\pi_{q^{0}}^{-1}-$ это обратное отображение к $\pi_{q_{0}}: \Lambda_{0} \rightarrow \mathbb{R}_{q^{0}}^{n}$. Таким образом, из (64) получаем

$$
\iint \Phi(q, t) F(q, p, t) d q d p=\int \kappa_{0}\left(q^{0}\right)\left[\left(\pi_{q_{0}}^{-1}\right)^{*} \widetilde{S}(t)\right]\left(q^{0}\right) d q^{0}=\int_{\Lambda_{0}} \widetilde{S}(t) d \mu_{0}
$$

В последнем равенстве мы использовали формулу $d \mu_{0}=\pi_{q^{0}}^{*}\left(\kappa\left(q^{0}\right) d q^{0}\right)$, которая следует из (60). Остается заметить, что интеграл $\int_{\Lambda_{0}} \widetilde{S}(t) d \mu_{0}$ совпадает с энтропией $H_{0}(t)$, заданной формулой (59). Утверждение доказано.

В заключение раздела мы укажем на одно естественное обобщение рассмотренного уравнения Власова-Лиувилля. Это обобщение связано с тем, что отображение Гамильтона $H[f, v]$ может быть определено на функциях $f$ и $v$, явно зависящих от времени,

$$
H[f, v](z, t)=H_{0}(z, t)+\int_{0}^{t} d \tau \int V\left(z, t, v\left(z^{\prime}, \tau\right), \tau\right) f\left(z^{\prime}, \tau\right) d z^{\prime} .
$$

Такому отображению отвечают уравнение Власова-Лиувилля

$$
\frac{\partial F}{\partial t}\left\langle J \frac{\partial H[F, \mathrm{id}]}{\partial z}(z, t), \frac{\partial F}{\partial z}\right\rangle=0
$$

и уравнение Власова-Гамильтона

$$
\dot{Z}(w, t)=J \frac{\partial H\left[F_{0}, Z\right]}{\partial z}(Z(w, t), t) .
$$


Развитая здесь теория может быть почти автоматически перенесена на отображения $H$ вида (66) или, более общим образом, на такие отображения, которые инвариантны относительно канонических преобразований и удовлетворяют условию $\frac{\delta H[f, v]}{\delta v\left(z^{\prime}, t^{\prime}\right)}(z, t)=0$ при $t^{\prime}>t$, т.е. зависят от значений функции $v$ лишь в моменты времени, предшествующие $t$.

\section{4. ОБ УРАВНЕНИИ ВЛАСОВА И КОЛЛЕКТИВНЫХ КОЛЕБАНИЯХ}

Уравнения среднего поля, или уравнения самосогласованного поля, в квантовой теории были использованы на 10 лет раньше, чем в классической. Никто не сделал перехода при $h \rightarrow 0$. И лишь через 10 лет их применил Власов для описания плазмы. Он установил роль уравнений в вариациях, определяющих так называемые коллективные колебания плазмы. Это было одним из основных его достижений.

При рассмотрении задачи многих тел имеют место два аспекта. Эти многие тела могут находиться в нашем трехмерном пространстве и как-то двигаться, может быть, сталкиваться, воздействовать друг на друга. Но мы можем рассматривать их и по-другому. А именно, можем рассматривать эти $N$ тел как одну точку (под $N$ телами мы понимаем $N$ материальных точек) в $3 N$-мерном пространстве. И в этом $3 N$-мерном пространстве эта одна точка совершает некоторое движение, перемещается, а мы можем следить за этим перемещением. В некоторых случаях оказывается полезен один из этих двух подходов, а в других случаях - другой. Так, с точки зрения математики проще рассматривать одну точку. А с точки зрения физики, так как мы живем все-таки в трехмерном пространстве, хотелось бы понимать, как материальные точки ведут себя в нашем трехмерном пространстве. Это более естественная картина. Можно, например, следить в трехмерном пространстве за одной частицей, которая находится в поле всех других частиц. В частности, на этом основана теория самосогласованного поля Власова, в которой считается, что все частицы примерно одинаковы и имеют одинаковое распределение. Можно считать, что одна частица как бы взаимодействует с другой, а та другая - это та же самая частица, т.е. распределена она точно так же, как и первая.

С математической точки зрения, важно было бы не интуитивное, а точное уравнение в трехмерном пространстве, которое бы соответствовало уравнению движения частицы в $3 N$-мерном пространстве. Оказывается, это можно сделать не интуитивно, как Власов, а точно с помощью вторичного квантования, т.е., иначе говоря, рассматривать поведение в трехмерном пространстве не функций, а операторов операторов рождения и уничтожения. Впервые это было сделано Дираком для квантовой механики. Однако рассматривать именно квантовую механику совершенно необязательно. Так, Шенберг рассматривал классическую механику и применил этот метод вторичного квантования к классическим объектам и к классической статистической физике.

Современный вывод уравнения Власова может быть сделан с помощью метода вторичного квантования для классических частиц ${ }^{5)}$. При этом при $N \rightarrow \infty$ получа-

\footnotetext{
5) В этом выводе необходимо использовать принцип тождественности классических частиц. Это не вызывает ни малейшего возражения. А вот конденсат типа конденсата Бозе-Эйнштейна, ко-
} 
ется система [20]

$$
\begin{aligned}
& \dot{u}(p, q, t)=\left(\frac{\partial U}{\partial q} \frac{\partial}{\partial p}-p \frac{\partial}{\partial q}\right) u(p, q, t)+ \\
& \quad+\iint d p^{\prime} d q^{\prime} v\left(p^{\prime}, q^{\prime}, t\right)\left(\frac{\partial V\left(q, q^{\prime}\right)}{\partial q} \frac{\partial}{\partial p}+\frac{\partial V\left(q, q^{\prime}\right)}{\partial q^{\prime}} \frac{\partial}{\partial p^{\prime}}\right) u\left(p^{\prime}, q^{\prime}, t\right) u(p, q, t), \\
& \dot{v}(p, q, t)=\left(\frac{\partial U}{\partial q} \frac{\partial}{\partial p}-p \frac{\partial}{\partial q}\right) v(p, q, t)+ \\
& \quad+\iint d p^{\prime} d q^{\prime} u\left(p^{\prime}, q^{\prime}, t\right)\left(\frac{\partial V\left(q, q^{\prime}\right)}{\partial q} \frac{\partial}{\partial p}+\frac{\partial V\left(q, q^{\prime}\right)}{\partial q^{\prime}} \frac{\partial}{\partial p^{\prime}}\right) v\left(p^{\prime}, q^{\prime}, t\right) v(p, q, t),
\end{aligned}
$$

где $U\left(q_{i}\right)$ - внешнее поле, $V\left(q_{i}, q_{j}\right)$ - парное взаимодействие.

Если положить вместо $u$ и $v$ операторы рождения и уничтожения $\widehat{u}, \widehat{v}$ в пространстве Фока, то после такой замены система (67) будет эквивалентна $N$-частичной задаче для системы Ньютона. Именно проекция из пространства Фока в $3 N$-мерное пространство $N$ частиц даст в точности систему уравнений Ньютона. Отсюда следует, что "уравнением характеристик для них с точки зрения операторного подхода будет уравнение Власова" ([21], с. 10). Этот подход позволяет получить поправки к уравнению Власова и разложению $N$-частичных уравнений Ньютона по степеням $1 / N$.

Отметим, что подстановкой

$$
u(p, q, t)=\sqrt{\rho(p, q, t)} e^{i \pi(p, q, t)}, \quad v(p, q, t)=\sqrt{\rho(p, q, t)} e^{-i \pi(p, q, t)},
$$

где $\rho$ - функция распределения, система (67) может быть приведена к виду

$$
\begin{aligned}
\dot{\rho}(p, q, t)= & \left(\frac{\partial W^{t}}{\partial q} \frac{\partial}{\partial p}-p \frac{\partial}{\partial q}\right) \rho(p, q, t) \\
\dot{\pi}(p, q, t)= & \left(\frac{\partial W^{t}}{\partial q} \frac{\partial}{\partial p}-p \frac{\partial}{\partial q}\right) \pi(p, q, t)+ \\
& +\iint d p^{\prime} d q^{\prime} \frac{\partial V\left(q, q^{\prime}\right)}{\partial q^{\prime}} \frac{\partial \pi\left(p^{\prime}, q^{\prime}, t\right)}{\partial p^{\prime}} \rho\left(p^{\prime}, q^{\prime}, t\right),
\end{aligned}
$$

где

$$
W^{t}(q)=U(q)+\iint V\left(q, q^{\prime}\right) \rho\left(p^{\prime}, q^{\prime}, t\right) d p^{\prime} d q^{\prime}
$$

Первое уравнение системы (69) является уравнением Власова, которое используется в физике при изучении систем многих частиц; второе, новое уравнение является линейным по функции $\pi$. Функции $u$ и $v$ называются полуплотностью, а $\pi$ - фазой. Полуплотность играет существенную роль в квазиклассическом приближении волновой функции квантовой механики. Фаза $\pi$ играет роль, когда потенциал $v(x-y)$ имеет особенность, а решение имеет нить ветвления (вихревая нить). Тогда для существования однозначного решения задачи $(69)$ в стационарном случае $e^{i \pi(p, q, \omega)}$

торый возникает здесь с необходимостью, вызывает неприятие: в первом случае тождественность можно допустить, а во втором - непривычно. 
дает условия квантования для $\omega$ - квантование вихрей, имеющих место и в классических задачах, связанных с электромагнитным полем, где спин проявляет себя как классический объект (см. ниже).

Точное доказательство перехода при $N \rightarrow \infty$ изложено в монографии [20]. Отметим, что вывод уравнения Власова, сделанным Боголюбовым из цепочек Боголюбова-Борна-Грина-Кирквуда-Ивона, не совсем корректен математически, так как гипотеза сохранения хаоса, используемая им, не всегда верна.

\section{5. ЗАКЛЮЧЕНИЕ}

Для того чтобы отличить классическую теорию в современном ее понимании от квантовой, нужно несколько изменить привычную для физиков идеологию, которая заключается в том, что под классической теорией понимается вся та теория, которая существовала в XIX веке до появления квантовой теории. На самом же деле классическая теория - это та теория, которая получается из квантовой в пределе $h \rightarrow 0$.

Так, Фейнман правильно заметил, что спин - это явление классической механики. Действительно, так получается при строгом переходе из квантовой механики в классическую. Это аналогично тому, как поляризация лучей не исчезает при увеличении частоты и, следовательно, является свойством геометрической, а не волновой оптики, как принято думать из-за того, что открытие поляризации света произошло в результате открытия волновой оптики.

Рассмотрим "яму Лифшица" - одномерное уравнение Шредингера с потенциалом с симметричными относительно начала координат двумя впадинами [10]. Его собственные функции симметричны или антисимметричны относительно начала координат. При $h \rightarrow 0$ эта симметрия остается, а поскольку квадрат модуля собственной функции отвечает вероятности пребывания частицы во впадинах, то в пределе $h \rightarrow 0$, т.е. в классическом пределе, частица с энергией, меньшей, чем высота барьера между впадинами, оказывается сразу в двух впадинах, хотя классическая частица не должна проникать через барьер. Этот простой пример показывает изменение идеологии классической теории.

Для понимания этого парадокса нужно учесть, что симметрия должна быть очень точная и что стационарность состояния означает, что это состояние возникает в пределе "бесконечно большого" времени. Это же относится и к бозе-распределению в классической теории газов (рис. 4).

Теперь коснемся понятий "коллективных колебаний” в классической физике и "квазичастиц" в квантовой физике. В классической физике им отвечают уравнения Власова самосогласованного (или среднего) поля, а в квантовой - уравнение Хартри (или Хартри-Фока).

Уравнения в вариациях зависят от того, около каких решений исходного уравнения мы рассматриваем вариации. Например, в работах [22], [23] рассматриваются вариации около микроканонического распределения в эргодической конструкции, а в [24], [25] - около наноканонического распределения, сосредоточенного на инвариантном многообразии меньшей размерности, т.е. не на многообразии постоянной энергии, а например, на лагранжевом многообразии размерности, совпадающей с размерностью конфигурационного пространства. 
Отметим следующий важнейший момент. Решение уравнения в вариациях для уравнения Власова не совпадает с классическим пределом для уравнений в вариациях, отвечающих уравнениям среднего поля в квантовой теории.

Рассмотрим квантовое уравнение среднего поля вида

$$
i h \frac{\partial}{\partial t} \varphi^{t}(x)=\left(-\frac{h^{2}}{2 m} \Delta+W_{t}(x)\right) \varphi^{t}(x), \quad W_{t}(x)=U(x)+\int V(x, y)\left|\varphi^{t}(y)\right|^{2} d y,
$$

при начальном условии $\left.\varphi\right|_{t=0}=\varphi_{0}$, где $\varphi_{0}$ такое, что $\varphi_{0} \in W_{2}^{\infty}\left(\mathbb{R}^{\nu}\right), \int d x\left|\varphi_{0}(x)\right|^{2}=1$.

Для получения асимптотики типа комплексного ростка [21] следует рассмотреть систему, состоящую из уравнения Хартри (30) и уравнения, ему сопряженного. Затем рассмотреть для нее систему в вариациях и, наконец, заменить вариации $\delta \varphi$ и $\delta \varphi^{*}$ на независимые функции $F$ и $G$. Для функций $F$ и $G$ получаем следующую систему уравнений:

$$
\begin{aligned}
i \frac{\partial F^{t}(x)}{\partial t} & =\int d y\left(\frac{\delta^{2} H}{\delta \varphi^{*}(x) \delta \varphi(y)} F^{t}(y)+\frac{\delta^{2} H}{\delta \varphi^{*}(x) \delta^{*} \varphi(y)} G^{t}(y)\right), \\
-i \frac{\partial G^{t}(x)}{\partial t} & =\int d y\left(\frac{\delta^{2} H}{\delta \varphi(x) \delta \varphi(y)} F^{t}(y)+\frac{\delta^{2} H}{\delta \varphi(x) \delta^{*} \varphi(y)} G^{t}(y)\right) .
\end{aligned}
$$

Получение классических уравнений из квантовых достигается подстановкой вида $\varphi=\chi e^{i S / h}$ (метод ВКБ), $\varphi^{*}=\chi^{*} e^{i S^{*} / h}, S=S^{*}, \chi=\chi(x, t) \in C^{\infty}, S=S(x, t) \in C^{\infty}$.

Для уравнения в вариациях естественно варьировать не только предельное уравнение для $\chi$ и $\chi^{*}$, но и функции $S$ и $S^{*}$. Это дает новый важный член решения уравнения для коллективных колебаний.

Проследим этот факт на простейшем примере, который исследуется в знаменитой работе Боголюбова [26].

Пусть $U=0$ в уравнении (70) в трехмерном ящике с длиной ребра $L$, при этом на волновые функции наложено условие периодичности (т.е. на торе с образующими $L, L, L)$. Тогда функция

$$
\varphi(x)=L^{-3 / 2} e^{\frac{i}{h}(p x-\Omega t)},
$$

где $p=2 \pi n / L, n$ - целочисленный вектор, удовлетворяет уравнению (70) при

$$
\Omega=\frac{p^{2}}{2 m}+L^{-3} \int V(x) d x .
$$

Рассмотрим функции $F^{(\lambda)}(x)$ и $G^{(\lambda)}(x)$, где $\lambda=2 \pi n / L, n$ - целочисленный вектор, $n \neq 0$, следующего вида:

$$
\begin{aligned}
& F^{(\lambda) t}(x)=L^{-3 / 2} \rho_{\lambda} e^{\frac{i}{h}|(p+\lambda) x+(\beta-\Omega) t|} \\
& G^{(\lambda) t}(x)=L^{-3 / 2} \sigma_{\lambda} e^{\frac{i}{h}|(-p+\lambda) x+(\beta+\Omega) t|},
\end{aligned}
$$

где

$$
\begin{gathered}
-\beta_{\lambda} \rho_{\lambda}=\left(\frac{(p+\lambda)^{2}}{2 m}-\frac{p^{2}}{2 m}+\widetilde{V}_{\lambda}\right) \rho_{\lambda}+V_{\lambda} \sigma_{\lambda}, \\
\beta_{\lambda} \rho_{\lambda}=\left(\frac{(p-\lambda)^{2}}{2 m}-\frac{p^{2}}{2 m}+\widetilde{V}_{\lambda}\right) \sigma_{\lambda}+V_{\lambda} \rho_{\lambda}, \\
\left|\sigma_{\lambda}\right|^{2}-\left|\rho_{\lambda}\right|^{2}=1, \quad \widetilde{V}_{\lambda}=L^{-3} \int V(x) e^{\frac{i}{h} \lambda x} d x .
\end{gathered}
$$


Из системы (75) находим

$$
\beta_{\lambda}=-p \lambda+\sqrt{\left(\frac{\lambda^{2}}{2 m}+\tilde{V}_{\lambda}\right)^{2}-\tilde{V}_{\lambda}^{2}} .
$$

В этом примере $u=e^{i s(x, t) / h}, u^{*}=e^{-s(x, t) / h}$, где $s(x, t)=p x+\beta t$, а вариация действия для вектора $\left(\delta u, \delta u^{*}\right)$ равна $\lambda x \pm \Omega t$. При более тщательном переходе к пределу $\widetilde{V}_{\lambda} \rightarrow V_{0}=L^{-3} \int V(x) d x$.

Таким образом, в классическом пределе мы получаем знаменитое соотношение Боголюбова (76). В рассмотренном случае $u(x)=0$, как и в линейном уравнении Шредингера, точное решение совпадает с квазиклассическим. В работе [27] исследуется случай $u(x) \neq 0$, и оказывается, что соотношение, аналогичное $(76)$, является классическим пределом при $h \rightarrow 0$ для уравнения в вариациях в этом общем случае. Кривая зависимости $\beta_{\lambda}$ от $\lambda$ называется кривой Ландау и определяет сверхтекучее состояние. Значение $\lambda_{\text {кр }}$, при котором сверхтекучесть пропадает, называется критерием Ландау.

Спектр, определяемый при $\lambda<\lambda_{\text {кр }}$, имеет отвечающий ему положительный спектр уравнения в вариациях. Это означает его метастабильность (см. [20]).

Поскольку Боголюбов рассматривал задачу без внешнего поля, то полученная им асимптотика совпала с квазиклассической во внешнем поле [24], [25]. Более того, это, по существу, и классический предел, так как параметр $h^{2}$ в работе Боголюбова [26] может быть компенсирован большим параметром $k$, что и сделано выше. Это становится особенно очевидно, если положить $\widetilde{V}_{\Lambda}=0$. Тогда получится идеальный газ, а он в данном случае может рассматриваться как классический. Именно поэтому автором доказано, что классические жидкости в нанотрубках обладают сверхтекучестью (см. [28], [29]), что и подтвердилось серией экспериментов (см. ссылки в работах [28]).

Благодарности. Работа выполнена при поддержке Программы фундаментальных исследований Президиума РАН № 27.

\section{Список литературы}

[1] В. П. Маслов, ТМФ, 161:2 (2009), 224-242.

[2] Б. Д. Сумм, Основы коллоидной химии, Издательский центр “Академия", М., 2007.

[3] E. A. Guggenheim, J. Chem. Phys., 13:7 (1945), 253-261.

[4] V.P. Maslov, Math. Notes, 86:4 (2009), 522-529.

[5] Л. Д. Ландау, Е. М. Лифшиц, Теоретическая физика Т. 5. Статистическая физика. Часть 1, Наука, М., 1964.

[6] V.P. Maslov, "Dequantization, Statistical Mechanics and Econophysics", Contemporary mathematics, Tropical and Idempotent Mathematics, 495, eds. G. L. Litvinov, S. N. Sergeev, AMS, 2009, 239-279.

[7] V.P. Maslov, Mathematical conception of the gas theory, arXiv: 0812.4669.

[8] А.И. Бурштейн, Молекулярная физика, Наука, Новосибирск, 1986.

[9] V.P. Maslov, Math. Notes, 86:1-2 (2009), 3-9.

[10] Л. Д. Ландау, Е. М. Лифшиц, Теоретическая физика. Т. 3. Квантовая механика. Нерелятивистская теория, Наука, М., 1974.

[11] В.П. Маслов, ТМФ, 159:2 (2009), 318-320.

[12] V.P. Maslov, Russ. J. Math. Phys., 16:1 (2009), 103-120. 
[13] V.P. Maslov, Threshold levels in Economics, arXiv: 0903.4783.

[14] V.P. Maslov, Math. Notes, 85:3 (2009), 305-321.

[15] Н. Н. Боголюбов, Изв. АН СССР. Сер. физ., 11:1 (1947), 77-90.

[16] Л. С. Понтрягин, А. А. Андронов, А. А. Витт, ЖЭЭТ, 3:3 (1933), 165-180.

[17] В. П. Маслов, Комплексные марковские иепи и континуальный интеграл Фейнмана, Наука, М., 1976.

[18] Г. Ламб, Гидродинамика, ОГИЗ, М.-Л., 1947.

[19] В. П. Маслов, Операторные методы, Наука, М., 1973.

[20] В. П. Маслов, О. Ю. Шведов, Метод комплексного ростка в многочастичных задачах и задачах квантовой теории поля, УРСС, М., 2000.

[21] В. П. Маслов, Комплексный метод ВКБ в нелинейных уравнениях, Наука, М., 1977.

[22] V.P. Maslov, Russ. J. Math. Phys., 3:4 (1995), 529-534.

[23] В. П. Маслов, А. С. Мищенко, Матем. сб., 189:6 (1998), 85-116.

[24] V.P. Maslov, Russ. J. Math. Phys., 2:4 (1994), 527-534.

[25] V.P. Maslov, Russ. J. Math. Phys., 3:1 (1995), 123-132.

[26] Н.Н. Боголюбов, "К теории сверхтекучести", Избранные труды в трех томах, т. 2, Наукова думка, Киев, 1970, 210-224; N. Bogolubov, Acad. Sci. USSR. J. Phys., 11 (1947), $23-32$.

[27] V.P. Maslov, Russ. J. Math. Phys., 3:3 (1995), 401-406.

[28] V.P. Maslov, Russ. J. Math. Phys., 14:3 (2007), 304-318; 14:4, 453-464; 15:1 (2008), 98-101; 15:2, 280-290.

[29] В. П. Маслов, ТМФ, 153:3 (2007), 388-408.

Поступила в редакцию 6.09.2009 